\title{
Semilattice Modes II: the amalgamation property *
}

\author{
Keith A. Kearnes ${ }^{\dagger}$
}

\begin{abstract}
Let $\mathcal{V}$ be a variety of semilattice modes with associated semiring $\mathbf{R}$. We prove that if $\mathbf{R}$ is a bounded distributive lattice, then $\mathcal{V}$ has the amalgamation property. We show that the converse is true when $\mathcal{V}$ is locally finite.
\end{abstract}

\section{Introduction}

The paper [3] analyzes the structure of semilattice modes and shows that, in many ways, semilattice modes are very similar to varieties of modules. We prove results in [3] which show that a number of the categorical and algebraic properties of varieties of modules are shared by varieties of semilattice modes. Most importantly, there is a commutative semiring associated with a variety of semilattice modes and each subdirectly irreducible semilattice mode is polynomially equivalent to a semimodule over the associated semiring.

In this paper, we point out a difference between varieties of modules and varieties of semilattice modes: all varieties of modules have the amalgamation property, but few varieties of semilattice modes have this property. Assume that $\mathcal{V}$ is a variety of semilattice modes and that $\mathbf{R}$ is the associated semiring. We show that if $\mathbf{R}$ is a bounded distributive lattice, then $\mathcal{V}$ has the amalgamation property. Conversely, if $\mathcal{V}$ is locally finite and has the amalgamation property, then $\mathbf{R}$ must be a bounded distributive lattice. We give an example to show that when $\mathcal{V}$ is not locally finite, then it is possible for $\mathcal{V}$ to have the amalgamation property even if $\mathbf{R}$ is not a bounded distributive lattice.

\section{Preliminaries}

We briefly review the material from [3] which will be needed in this paper. We expect that the reader will be familiar with the material in [5] and [6] to the degree that we need not review basic algebra or the definition of a mode. A semilattice mode is a mode which has a binary term operation which is a semilattice operation. If $\mathcal{V}$ is a variety of semilattice modes, then [3] proves that one can associate a semiring $\mathbf{R}(\mathcal{V})$ to $\mathcal{V}$ which determines most of the properties of $\mathcal{V}$. We explain now how $\mathbf{R}(\mathcal{V})$ is constructed.

*1991 Mathematical Subject Classification Primary 08B25.

${ }^{\dagger}$ Part of this paper was written while the author was supported by a fellowship from the Alexander von Humboldt Stiftung 
$\mathbf{F}_{\mathcal{V}}(x, y)$ denotes the free algebra on the set $\{x, y\}$ in the variety $\mathcal{V}$. Let $R$ be the subuniverse of $\mathbf{F}_{\mathcal{V}}(x, y)$ which is the image of the endomorphism determined by $x \mapsto x+y, y \mapsto y$. We define $\mathbf{R}(\mathcal{V})$ so that it has universe $R$ and operations $\cdot,+, 1,0$ which are defined as follows. For $s, t \in R$, we define $s \cdot t$ to be the element $e_{t}(s)$ where $e_{t}$ is the endomorphism of $\mathbf{F}_{\mathcal{V}}(x, y)$ determined by $x \mapsto t, y \mapsto y$. The operation + is just the semilattice operation of the variety (which is uniquely determined). + is an operation on $R$, since $R$ is a subuniverse of $\mathbf{F}_{\mathcal{V}}(x, y)$. 1 denotes the element $x+y \in R$ and 0 denotes the elements $y \in R$. The structure $\mathbf{R}(\mathcal{V})=\langle R ; \cdot,+, 1,0\rangle$ is called the semiring of $\mathcal{V}$.

Let us describe an alternate construction of $\mathbf{R}(\mathcal{V})$ which the reader will readily see agrees with the one above. This time we take for the universe of $\mathbf{R}(\mathcal{V})$ the set of all binary terms $r(x, y)$ of $\mathcal{V}$, modulo $\mathcal{V}$-equivalence, for which

$$
\mathcal{V} \models r(x, y)+y=r(x, y) .
$$

If $s$ and $t$ are such terms, then $s \cdot t:=s(t(x, y), y)$ is another, as is $s+t:=s(x, y)+t(x, y)$, $1:=x+y$ and $0:=y$. The collection of these binary terms under the described operations forms a semiring isomorphic to the one in the preceding paragraph. Here is the isomorphism: for a binary term $r$, take its interpretation in $\mathbf{F}=\mathbf{F}_{\mathcal{V}}(x, y)$ and apply $r^{\mathbf{F}}$ to the generators $x$ and $y$. The assignment $r \mapsto r^{\mathbf{F}}(x, y)$ is easily seen to be a bijection from the set of binary terms $r$ (modulo $\mathcal{V}$-equivalence) satisfying $r(x, y)+y=r(x, y)$ onto the set $R$. The operations can easily be seen to correspond.

Let $t\left(x_{0}, \ldots, x_{n-1}\right)$ be a term of $\mathcal{V}$. For each $i<n$, we define $\hat{t}_{i}(x, y)$ to be $t(y, \ldots, y, x, y, \ldots, y)+y$. For each $i<n$, the element $\hat{t}_{i}=\hat{t}_{i}(x, y)$ may be construed to be an element of $\mathbf{R}(\mathcal{V})$, according to the second construction of this semiring. We call $\hat{t}_{i}$ the $\mathbf{i}^{\text {th }}$ coefficient of $t$. We call

$$
\hat{t}_{0} \bullet x_{0}+\cdots+\hat{t}_{n-1} \bullet x_{n-1}
$$

the coefficient representation for $t$. The point of making these definitions is that coefficient representations may be manipulated in all the ways one manipulates such expressions for the terms in a variety of modules. (See Lemma 3.5 of [3].) Hence, all terms of $\mathcal{V}$ may be thought of as "linear combinations of variables" where the coefficients come from $\mathbf{R}(\mathcal{V})$. This motivates us to investigate the properties of $\mathbf{R}(\mathcal{V})$. The identities satisfied by $\mathbf{R}(\mathcal{V})$ are described in the next theorem which follows from Theorem 4.11 of [3].

THEOREM 2.1 The identities of $\mathbf{R}(\mathcal{V})$ may be summarized as follows:

- $\langle R ; \cdot, 1,0\rangle$ and $\langle R ;+, 0,1\rangle$ are commutative semigroups whose first constant is an identity element and whose second constant is a zero element for the semigroup operation.

- Multiplication $(\cdot)$ distributes over addition $(+)$.

- $\mathbf{R}(\mathcal{V}) \models r+r=r$.

(When we say "the identities of $\mathbf{R}(\mathcal{V})$ ", we mean that the given identities must hold in any semiring of the form $\mathbf{R}(\mathcal{V})$ and conversely that any semiring satisfying the listed identities arises as $\mathbf{R}(\mathcal{V})$ for some variety $\mathcal{V}$ of semilattice modes.) It is easy to show 
that $\mathbf{R}(\mathcal{V})$ is a bounded distributive lattice if and only if $\mathbf{R}(\mathcal{V}) \models r \cdot r=r$ in addition to the identities listed in the theorem.

It is shown in [3] that $\mathbf{R}(\mathcal{V})$, the semiring of coefficients of terms, is generated as a semiring by the coefficients of the basic operations. As a result, we have the following (weak) version of Lemma 4.14 of [3]:

THEOREM 2.2 If $\mathcal{V}$ is a variety of semilattice modes of finite type, then $\mathbf{R}(\mathcal{V})$ is a finitely generated semiring.

The structure of subdirectly irreducible algebras plays a big role in [3] and will also play a big role in this paper. The following example is basic.

Example 1. Let $\mathbf{S}=\langle S ;+\rangle$ be an algebra satisfying:

(a) $\mathbf{S}$ is a join-semilattice.

(b) $\mathbf{S}$ has a least element 0.

(c) $\mathbf{S}$ has an element $u$ which is the least element in $S-\{0\}$.

Let $U$ be a set of endomorphisms of $\mathbf{S}$ satisfying the following conditions:

(i) $U$ is closed under composition and contains the identity endomorphism.

(ii) Members of $U$ commute.

(iii) All members of $U$ are decreasing in the sense that if $f \in U$, then $f(x) \leq x$ (meaning $f(x)+x=x$ ).

(iv) If $x<y$ in $\mathbf{S}$, then there is an $f \in U$ such that $f(x)=0<f(y)$.

Define $\mathbf{S}(U)$ to be the algebra $\left\langle S ; b_{f}(f \in U)\right\rangle$ where $b_{f}(x, y)=f(x)+y$. The following result is Theorem 3.3 of [3]:

THEOREM 2.3 $\mathbf{A}$ is a subdirectly irreducible semilattice mode iff $\mathbf{A}$ is term equivalent to an algebra of the form $\mathbf{S}(U)$ where $\mathbf{S}$ satisfies $(a)-(c)$ and $U$ satisfies $(i)-(i v)$ of Example 1.

If $\mathbf{A}$ is a subdirectly irreducible semilattice mode, then $\mathbf{S}$ can always be taken to be the underlying semilattice of $\mathbf{A}$. $U$ can be taken to be the following set of functions:

$$
\begin{aligned}
U & =\left\{s^{\mathbf{A}}(x, 0) \mid s \text { is a binary term }\right\} \\
& =\left\{p(x) \in \mathrm{Pol}_{1} \mathbf{A} \mid p(0)=0\right\} \\
& =\left\{p(x) \in \mathrm{Pol}_{1} \mathbf{A} \mid 0 \in p(A)\right\}
\end{aligned}
$$

The equality of these sets implies that for any $p \in \mathrm{Pol}_{1} \mathbf{A}$ the following conditions are equivalent:

- $0 \in p(A)$.

- $p(0)=0$.

- $p(x)=s^{\mathbf{A}}(x, 0)$ for some binary term $s$. 
In fact, it is proved in Theorem 3.1 of [3] that these conditions are equivalent not just for subdirectly irreducible algebras, but for any semilattice mode which has an element 0 which is a least element in the semilattice order. From Theorem 2.3, each subdirectly irreducible has a least element 0 and a second least element $u$ and the monolith is the equivalence relation with $\{0, u\}$ as its only nontrivial block.

A very important semilattice mode can be constructed from $\mathbf{R}(\mathcal{V})$. Call a subset $I \subseteq$ $R(\mathcal{V})$ and annihilator ideal if $I$ is nonempty, closed under + , and closed downward. Let $\mathcal{I}$ be the set of annihilator ideals of $\mathbf{R}(\mathcal{V})$ and define $a \oplus b=a \cap b$ for $a, b \in \mathcal{I}$. For each $r \in \mathbf{R}(\mathcal{V})$ and each $a \in \mathcal{I}$ let $r^{-1}(a)=\{x \in R(\mathcal{V}) \mid r \cdot x \in a\}$. Then, $\mathbf{S}=\langle\mathcal{I} ; \oplus\rangle$ as a join-semilattice and $U=\left\{r^{-1}(x) \mid r \in R(\mathcal{V})\right\}$ is a collection of decreasing, commuting endomorphisms of $\mathbf{S}$.

If $f$ is an $n$-ary basic operation symbol for $\mathcal{V}$, then we define a corresponding $n$-ary operation $[f]$ on $\mathcal{I}$ by

$$
[f]\left(I_{0}, \ldots, I_{n-1}\right)=\hat{f}_{0}^{-1}\left(I_{0}\right) \oplus \cdots \oplus \hat{f}_{n-1}^{-1}\left(I_{n-1}\right)
$$

for $I_{j} \in \mathcal{I}, j<n$. Here $\hat{f}_{i}$ denotes $i^{\text {th }}$ coefficient of $f$, which is a member of $\mathbf{R}(\mathcal{V})$. We may equip $\mathcal{I}$ with operations $[f], f$ a basic operation symbol for $\mathcal{V}$, and obtain an algebra of the same type as $\mathcal{V}$. Let us write $\mathcal{I}(\mathcal{V})$ to denote this algebra. Theorems 4.16 and 4.17 of [3] together prove that:

THEOREM 2.4 $\mathcal{V}=\operatorname{SP}(\mathcal{I}(\mathcal{V}))$

From the way $\mathbf{R}(\mathcal{V})$ and $\mathcal{I}(\mathcal{V})$ are constructed, we see that this theorem has the following consequence.

COROLLARY 2.5 Let $\mathcal{V}$ be a variety of semilattice modes. The following conditions are equivalent.

(i) $\mathcal{V}$ is locally finite.

(ii) $\mathbf{R}(\mathcal{V})$ is finite.

(iii) $\mathcal{I}(\mathcal{V})$ is finite.

(iv) $\mathcal{V}=\mathrm{SP}(\mathbf{A})$ for a finite mode $\mathbf{A}$

Condition (iv) of this corollary may be stated as, " $\mathcal{V}$ has finitely many subdirectly irreducible members, all of which are finite."

Corollary 4.18 of [3] proves that if $\mathbf{A}$ is a finite subdirectly irreducible semilattice mode and $\mathcal{V}=V(\mathbf{A})$, then $\mathbf{A} \cong \mathcal{I}(\mathcal{V})$. We get the following result related to the previous corollary.

COROLLARY 2.6 If $\mathbf{A}$ is a finite subdirectly irreducible semilattice mode, then $\mathrm{V}(\mathbf{A})=\mathrm{SP}(\mathbf{A})$.

In the next section we shall need to know that a locally finite variety of semilattice modes has only finitely many subvarieties. This follows from Corollary 2.5 , but it also is a consequence of the following, more informative, result. (Theorem 4.20 of [3].)

THEOREM 2.7 If $\mathcal{V}$ is a variety of semilattice modes, then the lattice of equational theories extending the theory of $\mathcal{V}$ (and therefore the dual of the lattice of subvarieties) is isomorphic to $\operatorname{Con} \mathbf{R}(\mathcal{V})$. 


\section{The Amalgamation Property}

In this section, we classify the locally finite varieties of semilattice modes that have the amalgamation property (AP). The amalgamation property for $\mathcal{V}$ is the property that asserts that, whenever we have algebras $\mathbf{A}, \mathbf{B}, \mathbf{C} \in \mathcal{V}$ and embeddings $f_{1}: \mathbf{A} \rightarrow \mathbf{B}$ and $g_{1}: \mathbf{A} \rightarrow \mathbf{C}$, then we can find an algebra $\mathbf{D} \in \mathcal{V}$ and embeddings $f_{2}, g_{2}$ which complete a commutative diagram:

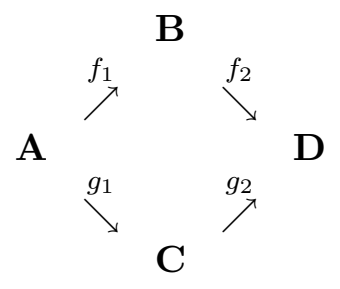

We say that the triple $\left(f_{2} ; g_{2} ; \mathbf{D}\right)$ completes the amalgam $\left(\mathbf{A} ; f_{1}, \mathbf{B} ; g_{1}, \mathbf{C}\right)$. When convenient, we shall assume that embeddings are inclusions.

We shall begin our proof that $\mathcal{V}$ has the AP when $\mathbf{R}(\mathcal{V})$ is a bounded distributive lattice by first proving it in the case where $\mathcal{V}$ is locally finite. A simple compactness argument then proves the result for non-locally finite $\mathcal{V}$. In our proof for the locally finite case, we consider amalgams of the form $\left(\mathbf{A} ; f_{1}, \mathbf{B} ; g_{1}, \mathbf{C}\right)$ where $|A|>1$, $\mathbf{B}$ is subdirectly irreducible and $g_{1}$ is essential. By Lemma 3.1 below, if we can complete these amalgams, then we can complete all amalgams. (A homomorphism $g_{1}: \mathbf{A} \rightarrow \mathbf{C}$ is essential if whenever $\theta \in$ Con $\mathbf{C}$ and $\theta>0_{\mathbf{C}}$, then $\left.\theta\right|_{\mathbf{A}} \stackrel{\text { def }}{=} g_{i}^{-1}(\theta)>0_{\mathbf{A}}$. We say that $\mathbf{B}$ is an essential extension of $\mathbf{A}$ if $\mathbf{A}$ is a embedded in $\mathbf{B}$ by an essential homomorphism.) Since $\mathcal{V}$ is locally finite, Corollary $2.5(i) \leftrightarrow(i v)$ proves $\mathcal{V}$ has a finite cardinality bound on the size of its subdirectly irreducible members; so $|B|$ is finite. It makes sense to talk about the height of an element in $\langle B ; \leq\rangle: b \in B$ has height $n$ if a shortest maximal chain from 0 to $b$ in $\langle B ; \leq\rangle$ has $n+1$ elements. We shall argue that $\left(\mathbf{A} ; f_{1}, \mathbf{B} ; g_{1}, \mathbf{C}\right)$ may be completed by using induction on $k$ where $k$ is the minimum height in $\langle B ; \leq\rangle$ of an element of the form $f_{1}(a), a \in A$.

The following lemma referred to in the last paragraph is proved in [2]. We will need the statement frequently in this paper and, in the proof of Theorem 3.7, we will need an understanding of the idea of the proof. To see that this theorem applies to varieties of semilattice modes, we refer the reader to Theorem 5.3 of [3] which proves that any variety of semilattice modes has the congruence extension property (CEP).

LEMMA 3.1 Assume that $\mathcal{V}$ has the CEP. Then $\mathcal{V}$ has the amalgamation property if and only if we can complete each amalgam $\left(\mathbf{A} ; f_{1}, \mathbf{B} ; g_{1}, \mathbf{C}\right)$ where $|A|>1, \mathbf{B}$ is subdirectly irreducible and $g_{1}$ is essential. When this is possible we can complete $\left(\mathbf{A} ; f_{1}, \mathbf{B} ; g_{1}, \mathbf{C}\right)$ with a triple $\left(f_{2} ; g_{2} ; \mathbf{D}\right)$ where $\mathbf{D}$ is subdirectly irreducible and $f_{2}$ is essential.

If we can complete all the amalgams $\left(\mathbf{A} ; f_{1}, \mathbf{B} ; g_{1}, \mathbf{C}\right)$ where all algebras are subdirectly irreducible and both maps are essential, then we can complete all amalgams in $\mathcal{V}$ where both maps are essential.

Proof: First, it is always possible to complete an amalgam $\left(\mathbf{A} ; f_{1}, \mathbf{B} ; g_{1}, \mathbf{C}\right)$ where $|A|=1$. Simply take $\mathbf{D}=\mathbf{B} \times \mathbf{C}$ and $f_{2}: \mathbf{B} \rightarrow \mathbf{D}: x \mapsto(x, a)$ where $A=\{a\}$. Similarly, take $g_{2}: \mathbf{C} \rightarrow \mathbf{D}: x \mapsto(a, x)$. Then $\left(f_{2} ; g_{2} ; \mathbf{D}\right)$ completes the amalgam. 
Suppose that can complete all amalgams where one of the homomorphisms is essential. We shall show how to complete an arbitrary amalgam $\left(\mathbf{A} ; f_{1}, \mathbf{B} ; g_{1}, \mathbf{C}\right)$. Let $\theta^{\mathbf{B}}$ be a congruence on $\mathbf{B}$ which is maximal among all $\theta \in$ Con $\mathbf{B}$ for which $\left.\theta\right|_{\mathbf{A}}=0_{\mathbf{A}}$. Then $\left(\mathbf{A} ; \bar{f}_{1}, \mathbf{B} / \theta^{\mathbf{B}} ; g_{1}, \mathbf{C}\right)$ is an amalgam where $\bar{f}_{1}$ is essential, so it can be completed; say that $\left(f_{2} ; g_{2} ; \mathbf{D}\right)$ completes the amalgam. Simlarly, we can find $\left(f_{3} ; g_{3} ; \mathbf{E}\right)$ which completes the amalgam $\left(\mathbf{A} ; f_{1}, \mathbf{B} ; \bar{g}_{1}, \mathbf{C} / \theta^{\mathbf{C}}\right)$. One easily verifies that

$$
\left(\left(f_{2} \times f_{3}\right) \circ\left(\bar{f}_{1} \times i d_{B}\right) ;\left(g_{2} \times g_{3}\right) \circ\left(i d_{C} \times \bar{g}_{1}\right) ; \mathbf{D} \times \mathbf{E}\right)
$$

completes the original amalgam: $\left(\mathbf{A} ; f_{1}, \mathbf{B} ; g_{1}, \mathbf{C}\right)$. Hence, to establish the amalgamation property we only need to show how to complete those amalgams $\left(\mathbf{A} ; f_{1}, \mathbf{B} ; g_{1}, \mathbf{C}\right)$ where one of the maps, say $g_{1}$, is essential.

Let $\mathbf{B} \leq \prod \mathbf{B} / \theta_{i}$ be a representation of $\mathbf{B}$ as a subdirect product of subdirectly irreducible algebras and define $\theta_{i}^{\mathbf{A}}=\left.\theta_{i}\right|_{\mathbf{A}}$ and $\theta_{i}^{\mathbf{C}}=$ a maximal congruence $\theta \in \operatorname{Con} \mathbf{C}$ such that $\left.\theta\right|_{\mathbf{A}}=\theta_{i}{ }^{\mathbf{A}}$ (there is at least one since $\mathcal{V}$ has the CEP). If we can complete each amalgam $\left(\mathbf{A} / \theta_{i}^{\mathbf{A}} ; f_{1}^{(i)}, \mathbf{B} / \theta_{i} ; g_{1}^{(i)}, \mathbf{C} / \theta_{i}^{\mathbf{C}}\right)$, then the product of the amalgamating algebras, $\left\{\mathbf{D}_{i} \mid i \in I\right\}$, together with the obvious maps (the restrictions of product maps) amalgamates $\mathbf{B}$ with $\mathbf{C} /\left(\cap \theta_{i}^{\mathbf{C}}\right)$ over $\mathbf{A} /\left(\cap \theta_{i}^{\mathbf{A}}\right)$. But $\cap \theta_{i}^{\mathbf{A}}=\left.\left(\cap \theta_{i}\right)\right|_{\mathbf{A}}=\left.0_{\mathbf{B}}\right|_{\mathbf{A}}=$ $0_{\mathbf{A}}$. Further, $\left.\left(\cap \theta_{i}^{\mathbf{C}}\right)\right|_{\mathbf{A}}=\cap \theta_{i}^{\mathbf{A}}=0_{\mathbf{A}}$. Since $g_{1}: \mathbf{A} \rightarrow \mathbf{C}$ is essential, we get that $\cap \theta_{i}^{\mathbf{C}}=$ $0_{\mathbf{C}}$. Therefore we even get that the product of the amalgamating algebras, $\left\{\mathbf{D}_{i} \mid i \in I\right\}$, with the obvious maps amalgamates $\mathbf{B}$ with $\mathbf{C}$ over $\mathbf{A}$. Hence, all amalgams can be completed iff we can complete those where $\mathbf{B}$ is subdirectly irreducible and $\mathbf{C}$ is an essential extension of the algebra $\mathbf{A}$.

Consider $\left(\mathbf{A} ; f_{1}, \mathbf{B} ; g_{1}, \mathbf{C}\right)$ where $\mathbf{B}$ is subdirectly irreducible and $g_{1}$ is essential. If $\left(f_{2} ; g_{2} ; \mathbf{D}\right)$ completes this amalgam, then we may choose $\psi \in$ Con $\mathbf{D}$ maximal with respect to the property that $\left.\psi\right|_{\mathbf{B}}=0_{\mathbf{B}}$. Then, $\left.\psi\right|_{\mathbf{A}}=0_{\mathbf{A}}$ and, since $g_{1}$ is essential, $\left.\psi\right|_{\mathbf{C}}=0_{\mathbf{C}}$. Thus $\left(\bar{f}_{2} ; \overline{g_{2}} ; \mathbf{D} / \psi\right)$ completes the amalgam with $\overline{f_{2}}$ essential and (consequently) with $\mathbf{D} / \psi$ subdirectly irreducible.

The argument for the second statement of this lemma is similar to the argument above. Choose a family $\left\{\theta_{i} \mid i \in I\right\}$ of strictly meet-irreducible congruences that separate the points of $\mathbf{A}$. Let $\theta_{i}^{\mathbf{B}}$ and $\theta_{i}^{\mathbf{C}}$ be maximal extensions of $\theta_{i}$ to $\mathbf{B}$ and $\mathbf{C}$ respectively. Necessarily, these congruences are strictly meet-irreducible and separate the points of $\mathbf{B}$ and $\mathbf{C}$ respectively (that they separate points follows from the fact that $\mathbf{B}$ and $\mathbf{C}$ are essential extensions of $\mathbf{A})$. If all the amalgams $\left(\mathbf{A} / \theta_{i} ; f_{1}^{(i)}, \mathbf{B} / \theta_{i}^{\mathbf{B}} ; g_{1}^{(i)}, \mathbf{C} / \theta_{i}^{\mathbf{C}}\right)$ can be completed, then taking products completes the original amalgam.

Now we begin a sequence of technical lemmas which show how to construct amalgamating triples $\left(f_{2} ; g_{2} ; \mathbf{D}\right)$.

LEMMA 3.2 If $\mathcal{V}$ is a variety of semilattice modes, $\mathbf{A} \in \mathcal{V}$ and $\mathbf{C}$ is a maximal essential extension of $\mathbf{A}$ in $\mathcal{V}$, then $0^{\mathbf{C}}$ exists and equals $\bigwedge_{a \in A} g_{1}(a)$. In particular, if $0^{\mathbf{A}}$ exists, then $g_{1}\left(0^{\mathbf{A}}\right)=0^{\mathbf{C}}$.

Proof: Let $\mathbf{C} \leq \prod_{i \in I} \mathbf{C}_{i}$ be a representation of $\mathbf{C}$ as a subdirect product of subdirectly irreducible semilattice modes. Choose $\theta \in \operatorname{Con} \prod_{i \in I} \mathbf{C}_{i}$ maximal with respect to the property that $\left.\theta\right|_{C}$ is the zero congruence of $\mathbf{C}$. Each $\mathbf{C}_{i}$ is subdirectly irreducible, so each has a least element in the semilattice order. Hence, $\Pi_{i \in I} \mathbf{C}_{i}$ has a least element, 
as does $\left(\Pi_{i \in I} \mathbf{C}_{i}\right) / \theta$. But the latter algebra is an essential extension of $\mathbf{C}$ and therefore is isomorphic to $\mathbf{C}$. It follows that $\mathbf{C}$ has a least element, $0^{\mathbf{C}}$, in the semilattice order.

To simplify the notation, assume that $A \subseteq C$ and that $g_{1}(x)=x$. We must show that $0^{\mathbf{C}}=\bigwedge_{a \in A} a$. To show this means that we must rule out the possibility that there is an element $c \in C-\left\{0^{\mathbf{C}}\right\}$ such that $c \leq a$ for all $a \in A$. Assume that $c$ is such an element. Then $\theta=\operatorname{Cg}^{\mathbf{C}}\left(0^{\mathbf{C}}, c\right)$ is a nonzero congruence of $\mathbf{C}$. Since $\mathbf{C}$ is an essential extension of $\mathbf{A},\left.\theta\right|_{A}>0_{\mathbf{A}}$. Assume that $\left.(a, b) \in \theta\right|_{A}-0_{\mathbf{A}}$. By Mal'cev's congruence generation theorem we can find a sequence of elements $a=x_{0}, x_{1}, \ldots, x_{n}=b$ such that for all $i<n$ we have $\left\{x_{i}, x_{i+1}\right\}=\left\{p_{i}(c), p_{i}\left(0^{\mathbf{C}}\right)\right\}$ for some $p_{i} \in \mathrm{Pol}_{1} \mathbf{C}$. We alter this to a new sequence

$$
a=y_{0}, \ldots, y_{n}=z_{0}, \ldots, z_{n}=b
$$

by defining $y_{i}=x_{0}+\cdots+x_{i}$ for $i \leq n$ and $z_{j}=x_{j}+\cdots+x_{n}$ for $j \leq n$. We further define $r_{i}(x)=p_{i}(x)+y_{i} \in \mathrm{Pol}_{1} \mathbf{C}$ and $s_{i}(x)=p_{i}(x)+z_{i+1} \in \operatorname{Pol}_{1} \mathbf{C}$ for $i<n$. The sequence of polynomials

$$
\left(r_{0}, \ldots, r_{n-1}, s_{0}, \ldots, s_{n-1}\right)
$$

witnesses the fact that

$$
a=y_{0}, \ldots, y_{n}=z_{0}, \ldots, z_{n}=b
$$

is a Mal'cev chain connecting $a$ to $b$ by polynomial images of $\left\{0^{\mathbf{C}}, c\right\}$. This chain has the further property that $y_{i} \leq y_{i+1}$ and $z_{i+1} \leq z_{i}$. By deleting unnecessary links in the chain, we may assume that these inequalities are strict and, because $a \neq b$, there remains at least one strict inequality. Without loss of generality, $y_{0}=a<y_{1}$. Now $\left\{r_{0}\left(0^{\mathbf{C}}\right), r_{0}(c)\right\}=\left\{a, y_{1}\right\}$. Since our assumption is that $c$ lies strictly below every element of $A$, we have $0^{\mathbf{C}}<c<a<y_{1}$. Hence $\left(r_{0}\left(0^{\mathbf{C}}\right), r_{0}(c)\right)=\left(a, y_{1}\right)$. Now suppose that $r_{0}(x)=t^{\mathbf{C}}(x, \bar{u}), \bar{u} \in C^{n}$. Then

$$
\begin{aligned}
r_{0}(x) & =t^{\mathbf{C}}(x, \bar{u}) \\
& =t^{\mathbf{C}}\left(x+0^{\mathbf{C}}, 0^{\mathbf{C}}+u_{0}, \ldots, 0^{\mathbf{C}}+u_{n-1}\right) \\
& =t^{\mathbf{C}}\left(x, 0^{\mathbf{C}}, \ldots, 0^{\mathbf{C}}\right)+t^{\mathbf{C}}\left(0^{\mathbf{C}}, u_{0}, \ldots, u_{n-1}\right) \\
& =t_{0}^{\mathbf{C}}\left(x, 0^{\mathbf{C}}\right)+r_{0}\left(0^{\mathbf{C}}\right) \\
& =t_{0}^{\mathbf{C}}\left(x, 0^{\mathbf{C}}\right)+a \\
& =t_{0}^{\mathbf{C}}\left(x, 0^{\mathbf{C}}\right)+t_{0}^{\mathbf{C}}(a, a) \\
& =t_{0}^{\mathbf{C}}(x+a, a) .
\end{aligned}
$$

In particular,

$$
a<y_{1}=r_{0}(c)=t_{0}^{\mathrm{C}}(c+a, a) \leq t_{0}^{\mathrm{C}}(a+a, a)=a
$$

which is the contradiction we sought. The lemma is proved.

LEMMA 3.3 Let $\mathcal{V}$ be a variety of semilattice modes. If $\left(\mathbf{A} ; f_{1}, \mathbf{B} ; g_{1}, \mathbf{C}\right)$ is an amalgam in $\mathcal{V}$ where

(i) $\mathbf{B}$ is subdirectly irreducible,

(ii) $g_{1}: \mathbf{A} \rightarrow \mathbf{C}$ is an essential embedding,

(iii) $0^{\mathbf{A}}$ exists,

(iv) $f_{1}\left(0^{\mathbf{A}}\right)=0^{\mathbf{B}}, g_{1}\left(0^{\mathbf{A}}\right)=0^{\mathbf{C}}$,

then it is possible to complete $\left(\mathbf{A} ; f_{1}, \mathbf{B} ; g_{1}, \mathbf{C}\right)$ in $\mathcal{V}$. 
Proof: The argument we use here is similar to the argument that proves that a variety of modules has the amalgamation property. We embed each of $\mathbf{B}$ and $\mathbf{C}$ into the product $\mathbf{B} \times \mathbf{C}$ and then factor by the least congruence that identifies the two images of $\mathbf{A}$.

Let $\mathbf{D}=(\mathbf{B} \times \mathbf{C}) / \theta$ where $\theta$ is the congruence on $\mathbf{B} \times \mathbf{C}$ generated by $G=$ $\left\{\left\langle\left(f_{1}(x), 0^{\mathbf{C}}\right),\left(0^{\mathbf{B}}, g_{1}(x)\right)\right\rangle \mid x \in A\right\}$. Define $f_{2}: \mathbf{B} \rightarrow \mathbf{D}$ by $f_{2}(b)=\left(b, 0^{\mathbf{C}}\right) / \theta$ and similarly define $g_{2}: \mathbf{C} \rightarrow \mathbf{D}$ by $g_{2}(c)=\left(0^{\mathbf{B}}, c\right) / \theta$. Clearly $\mathbf{D} \in \mathcal{V}$ and $f_{2} \circ f_{1}(a)=$ $\left(f_{1}(a), 0^{\mathbf{C}}\right) / \theta=\left(0^{\mathbf{B}}, g_{1}(a)\right) / \theta=g_{2} \circ g_{1}(a)$. The only thing we need to verify to conclude that $\left(f_{2} ; g_{2} ; \mathbf{D}\right)$ completes the amalgam $\left(\mathbf{A} ; f_{1}, \mathbf{B} ; g_{1}, \mathbf{C}\right)$ is that $f_{2}$ and $g_{2}$ are 1-1 homomorphisms.

Modes are idempotent, so the function

$$
\mathbf{B} \rightarrow \mathbf{B} \times \mathbf{C}: b \mapsto\left(b, 0^{\mathbf{C}}\right)
$$

is a 1-1 homomorphism. $f_{2}$ is just the composition of this homomorphism with the natural homomorphism $\mathbf{B} \times \mathbf{C} \rightarrow(\mathbf{B} \times \mathbf{C}) / \theta$. From this it follows that $f_{2}$ is a homomorphism. Similarly $g_{2}$ is a homomorphism.

Finally, we must prove that $f_{2}$ and $g_{2}$ are 1-1. The strategy here will be to show that if $g_{2}$ is not an embedding, then $f_{2}$ is not an embedding. Then we shall prove that $f_{2}$ is an embedding. This will finish the proof. Let us begin by factoring $g_{2}$ as $g_{2}=$ $n \circ g$ where

$$
g: \mathbf{C} \rightarrow \mathbf{B} \times \mathbf{C}: c \mapsto\left(0^{\mathbf{B}}, c\right)
$$

and $n: \mathbf{B} \times \mathbf{C} \rightarrow(\mathbf{B} \times \mathbf{C}) / \theta$ is the natural map. If $g_{2}$ is not an embedding, then there are distinct $c, d \in C$ such that $g(c) \theta g(d)$. This means that $g^{-1}(\theta)>0_{\mathbf{C}}$. As $g_{1}: \mathbf{A} \rightarrow \mathbf{C}$ is essential, $g_{1}^{-1}\left(g^{-1}(\theta)\right)>0_{\mathbf{A}}$. It is possible to find distinct $a, b \in A$ such that $\left(g \circ g_{1}(a), g \circ g_{1}(b)\right) \in \theta$. Written differently, $\left(\left(0^{\mathbf{B}}, g_{1}(a)\right),\left(0^{\mathbf{B}}, g_{1}(b)\right)\right) \in \theta$. But

$$
\left(f_{1}(a), 0^{\mathbf{C}}\right) \theta\left(0^{\mathbf{B}}, g_{1}(a)\right) \theta\left(0^{\mathbf{B}}, g_{1}(b)\right) \theta\left(f_{1}(b), 0^{\mathbf{C}}\right)
$$

from the way we defined $\theta$. Now let's factor $f_{2}$ as $f_{2}=n^{\prime} \circ f$ where

$$
f: \mathbf{B} \rightarrow \mathbf{B} \times \mathbf{C}: b \mapsto\left(b, 0^{\mathbf{C}}\right)
$$

and $n^{\prime}: \mathbf{B} \times \mathbf{C} \rightarrow(\mathbf{B} \times \mathbf{C}) / \theta$ is the natural map. We have $\left(f_{1}(a), f_{1}(b)\right) \in f^{-1}(\theta)-0_{\mathbf{B}}$. If $\mu$ denotes the monolith of $\mathbf{B}$, this implies that $\mu \subseteq f^{-1}(\theta)$. If $\mu=\mathrm{Cg}^{\mathbf{B}}\left(0^{\mathbf{B}}, u\right)$, then we get that $\left\langle\left(0^{\mathbf{B}}, 0^{\mathbf{C}}\right),\left(u, 0^{\mathbf{C}}\right)\right\rangle \in \theta$. There must be some unary polynomial $p \in \mathrm{Pol}_{1} \mathbf{B} \times \mathbf{C}$ and a pair $\langle u, v\rangle=\left\langle\left(f_{1}(w), 0^{\mathbf{C}}\right),\left(0^{\mathbf{B}}, g_{1}(w)\right)\right\rangle \in G$ such that

$$
\left(0^{\mathbf{B}}, 0^{\mathbf{C}}\right)=p(u) \neq p(v)
$$

or the same condition with $u$ and $v$ interchanged. (Recall that $G$ is the set of generators for $\theta$.) Assume that $p(x)=t^{\mathbf{B} \times \mathbf{C}}\left(x, \overline{\left(e_{i}, f_{i}\right)}\right)$ for some term $t$. Since $\left(0^{\mathbf{B}}, 0^{\mathbf{C}}\right)$ is a neutral element for + in $\mathbf{B} \times \mathbf{C}$, we get that

$$
\begin{aligned}
p(x) & =t^{\mathbf{B} \times \mathbf{C}}\left(x+\left(0^{\mathbf{B}}, 0^{\mathbf{C}}\right), \overline{\left(0^{\mathbf{B}}, 0^{\mathbf{C}}\right)+\left(e_{i}, f_{i}\right)}\right) \\
& =t^{\mathbf{B} \times \mathbf{C}}\left(x, \overline{\left(0^{\mathbf{B}}, 0^{\mathbf{C}}\right)}\right)+t^{\mathbf{B} \times \mathbf{C}}\left(\left(0^{\mathbf{B}}, 0^{\mathbf{C}}\right), \overline{\left(e_{i}, f_{i}\right)}\right) \\
& =t_{0}^{\mathbf{B} \times \mathbf{C}}\left(x,\left(0^{\mathbf{B}}, 0^{\mathbf{C}}\right)\right)+\left(0^{\mathbf{B}}, 0^{\mathbf{C}}\right) \\
& =t_{0}^{\mathbf{B} \times \mathbf{C}}\left(x,\left(0^{\mathbf{B}}, 0^{\mathbf{C}}\right)\right) .
\end{aligned}
$$


Here $t_{0}(x, y)=t(x, y, \ldots, y)$. Our argument used the fact that

$$
\left(0^{\mathbf{B}}, 0^{\mathbf{C}}\right) \leq t^{\mathbf{B} \times \mathbf{C}}\left(\left(0^{\mathbf{B}}, 0^{\mathbf{C}}\right), \overline{\left(e_{i}, f_{i}\right)}\right)=p\left(\left(0^{\mathbf{B}}, 0^{\mathbf{C}}\right)\right) \leq p(u)=\left(0^{\mathbf{B}}, 0^{\mathbf{C}}\right)
$$

(or the same statement with $v$ in place of $u$ ).

To summarize, we have either

$$
\left(0^{\mathbf{B}}, 0^{\mathbf{C}}\right)=t_{0}^{\mathbf{B} \times \mathbf{C}}\left(\left(f_{1}(w), 0^{\mathbf{C}}\right),\left(0^{\mathbf{B}}, 0^{\mathbf{C}}\right)\right)<t_{0}^{\mathbf{B} \times \mathbf{C}}\left(\left(0^{\mathbf{B}}, g_{1}(w)\right),\left(0^{\mathbf{B}}, 0^{\mathbf{C}}\right)\right)
$$

or else

$$
\left(0^{\mathbf{B}}, 0^{\mathbf{C}}\right)=t_{0}^{\mathbf{B} \times \mathbf{C}}\left(\left(0^{\mathbf{B}}, g_{1}(w)\right),\left(0^{\mathbf{B}}, 0^{\mathbf{C}}\right)\right)<t_{0}^{\mathbf{B} \times \mathbf{C}}\left(\left(f_{1}(w), 0^{\mathbf{C}}\right),\left(0^{\mathbf{B}}, 0^{\mathbf{C}}\right)\right) .
$$

Both cases lead to the same kind of contradiction. In the first case we have

$$
0^{\mathbf{B}}=t_{0}^{\mathbf{B}}\left(f_{1}(w), 0^{\mathbf{B}}\right) \leq t_{0}^{\mathbf{B}}\left(0^{\mathbf{B}}, 0^{\mathbf{B}}\right)=0^{\mathbf{B}}
$$

in the first coordinate, so

$$
f_{1}\left(0^{\mathbf{A}}\right)=0^{\mathbf{B}}=t_{0}^{\mathbf{B}}\left(f_{1}(w), f_{1}\left(0^{\mathbf{A}}\right)\right)=f_{1}\left(t_{0}^{\mathbf{A}}\left(w, 0^{\mathbf{A}}\right)\right) .
$$

Since $f_{1}$ is 1-1, we get that $t_{0}^{\mathbf{A}}\left(w, 0^{\mathbf{A}}\right)=0^{\mathbf{A}}$. Now in the second coordinate we must have

$$
{ }_{0}^{\mathbf{C}}=t_{0}^{\mathbf{C}}\left(0^{\mathbf{C}}, 0^{\mathbf{C}}\right)<t_{0}^{\mathbf{C}}\left(g_{1}(w), 0^{\mathbf{C}}\right)
$$

and since $g_{1}$ is 1-1 we get in the same way that $0^{\mathbf{A}}<t_{0}^{\mathbf{A}}\left(w, 0^{\mathbf{A}}\right)\left(=0^{\mathbf{A}}\right)$. This is a contradiction. The second case (described in the second displayed line of this paragraph) can be handled by the same argument.

COROLLARY 3.4 Let $\mathcal{V}$ be a variety of semilattice modes. It is possible to complete all amalgams $\left(\mathbf{A} ; f_{1}, \mathbf{B} ; g_{1}, \mathbf{C}\right)$ in $\mathcal{V}$ where $f_{1}$ and $g_{1}$ are essential.

Proof: By Lemma 3.1, it suffices to consider only the case where $\mathbf{A}, \mathbf{B}$ and $\mathbf{C}$ are subdirectly irreducible. Saying that $f_{1}$ and $g_{1}$ are essential means that

$$
f_{1}\left(0^{\mathbf{A}}\right)=0^{\mathbf{B}}, g_{1}\left(0^{\mathbf{A}}\right)=0^{\mathbf{C}} \text { and } f_{1}\left(u^{\mathbf{A}}\right)=u^{\mathbf{B}}, g_{1}\left(u^{\mathbf{A}}\right)=u^{\mathbf{C}} .
$$

In particular, the hypotheses of Lemma 3.3 are satisfied, so $\left(\mathbf{A} ; f_{1}, \mathbf{B} ; g_{1}, \mathbf{C}\right)$ can be completed.

The next lemma is a crucial part of our argument. It is the place where we require that $\mathbf{R}(\mathcal{V})$ be a finite, bounded distributive lattice. The point of this lemma is to show how a subdirectly irreducible $\mathbf{T}$ with monolith $\mu$ can be reconstructed from $\mathbf{T} / \mu$ and the subalgebra generated by the unique nontrivial $\mu$-class.

LEMMA 3.5 Assume that $\mathcal{V}$ is a variety of semilattice modes with $\mathbf{R}(\mathcal{V})$ a finite, bounded distributive lattice. Let $\mathbf{T} \in \mathcal{V}$ be a subdirectly irreducible semilattice mode with monolith $\mu=\operatorname{Cg}^{\mathbf{T}}(0, u), 0<u$. Set $\mathbf{V}$ equal to the image of the endomorphism $\mathbf{T} \rightarrow \mathbf{T}: x \mapsto x+u$ and $\mathbf{W}=\operatorname{Sg}^{\mathbf{T}}(\{0, u\})$. Then $\mathbf{V} \cong \mathbf{T} / \mu$ and the following conditions hold. 
(i) For any binary term $b(x, y)$ we have

$$
\forall x \in V\left(b^{\mathbf{W}}(u, 0)=u \rightarrow b^{\mathbf{V}}\left(x, 0^{\mathbf{V}}\right)=x\right) .
$$

(ii) There is a term $c(x, y)$ such that

$$
c^{\mathbf{W}}(u, 0)=0 \quad \text { and } \quad \forall x \in V\left(c^{\mathbf{V}}\left(x, 0^{\mathbf{V}}\right)=x\right) .
$$

(iii) Whenever $e<f$ in $\mathbf{V}$ there is a term $b(x, y)$ such that $b^{\mathbf{V}}\left(e, 0^{\mathbf{V}}\right)=0^{\mathbf{V}}<$ $b^{\mathbf{V}}\left(f, 0^{\mathbf{V}}\right)$.

Conversely, assume that $\mathbf{W}$ is a 2-element semilattice mode with universe $\left\{0 \mathbf{W}, u^{\mathbf{W}}\right\}$ and that $\mathbf{V}$ is a semilattice mode of the same type which has a least element $0 \mathbf{V}$ and $\mathbf{V}$ and $\mathbf{W}$ are related as in conditions $(i)-($ iii $)$. If $\theta$ is the congruence on $\mathbf{V} \times \mathbf{W}$ generated by

$$
G=\left\{\left\langle\left(c, 0^{\mathbf{W}}\right),\left(c, u^{\mathbf{W}}\right)\right\rangle \mid c \in V-\left\{0^{\mathbf{V}}\right\}\right\},
$$

then $\mathbf{T}^{\prime}:=(\mathbf{V} \times \mathbf{W}) / \theta$ is a subdirectly irreducible with monolith $\mu=\operatorname{Cg}\left(0^{\mathbf{T}^{\prime}}, u^{\mathbf{T}^{\prime}}\right)$ such that $\mathbf{V} \cong \mathbf{T}^{\prime} / \mu$ and $\mathbf{W} \cong \operatorname{Sg}^{\mathbf{T}^{\prime}}\left(\left\{0^{\mathbf{T}^{\prime}}, u^{\mathbf{T}^{\prime}}\right\}\right)$.

Finally, if $\mathbf{T} \in \mathcal{V}$ is subdirectly irreducible, $\mathbf{V}$ and $\mathbf{W}$ are related to $\mathbf{T}$ as in the first part of this lemma and $\mathbf{T}^{\prime}=(\mathbf{V} \times \mathbf{W}) / \theta$, then the function

$$
f: \mathbf{T}^{\prime} \rightarrow \mathbf{T}:(v, w) / \theta \mapsto \begin{cases}v & \text { if } v>0 \mathbf{V} \\ w & \text { if } v=0\end{cases}
$$

is an isomorphism.

Proof: Before starting the proof, we explain how we intend to use the hypothesis that $\mathbf{R}(\mathcal{V})$ is a bounded distributive lattice. Choose an algebra $\mathbf{A} \in \mathcal{V}$ which has a least element 0 . Pick a polynomial $p \in \mathrm{Pol}_{1} \mathbf{A}$ such that $0 \in p(A)$. By the remarks following Theorem 2.3, there is a binary term $s(x, y)$ such that $p(x)=s^{\mathbf{A}}(x, 0)$. The term $t(x, y):=s(x, y)+y$ has $t^{\mathbf{A}}(x, 0)=s^{\mathbf{A}}(x, 0)$. Replacing $s$ with $t$ if necessary, we may assume that $s \in R(\mathcal{V})$. The assumption that $\mathbf{R}(\mathcal{V})$ is a bounded distributive lattice is equivalent to $\mathbf{R}(\mathcal{V}) \models r^{2}=r$, so we have

$$
\mathcal{V} \models s(s(x, y), y)=s(x, y) .
$$

In particular, this forces $p(x)=s^{\mathbf{A}}(x, 0)$ to be an idempotent unary polynomial. To summarize, if $0 \in p(A)$, then we have $p(p(x))=p(x)$ for all $x \in A$.

Now we begin the proof. In the first claim, $\mathbf{V} \cong \mathbf{T} / \mu$ since the polynomial $x \mapsto x+u$ is an endomorphism of $\mathbf{T}$ with kernel $\mu$ and image $\mathbf{V}$.

To prove $(i)$, we first note that if $p \in \operatorname{Pol}_{1} \mathbf{T}$, then $p(\mu) \subseteq 0 \mathbf{T}$ or else $p(x)=x$ for all $x \in T$. This is proved for any subdirectly irreducible semilattice mode in Theorem 3.2 of [3], but we give a short proof here that works for our mode $\mathbf{T}$. Since $\mathbf{T}$ is a mode and $p$ is a polynomial of $\mathbf{T}, p$ is an endomorphism of $\mathbf{T}$. The monolith of $\mathbf{T}$ is $\mu=\mathrm{Cg}^{\mathbf{T}}(0, u)$, which has $\{0, u\}$ as its only nontrivial block, so $p(\mu) \nsubseteq 0_{\mathbf{T}}$ implies that $p$ is a 1-1 endomorphism for which

$$
p(\{0, u\})=\{0, u\} .
$$

Therefore, $p$ has 0 in its range, so $p(p(x))=p(x)$ for all $x \in T$. The only idempotent, 1-1 endomorphism of $\mathbf{T}$ is the identity, so $p(\mu) \nsubseteq \subseteq \mathbf{T}$ implies that $p(x)=x$ for all 
$x \in T$. We use this observation in the proof of $(i)$ as follows: If $b^{\mathbf{W}}(u, 0)=u$, then $b^{\mathbf{T}}(\mu, 0) \nsubseteq 0_{T}$, so $b^{\mathbf{T}}(x, 0)=x$ for all $x \in T$. $\mathbf{V}$ is a homomorphic image of $\mathbf{T}$, so $b^{\mathbf{V}}\left(x, 0^{\mathbf{V}}\right)=x$ for all $x \in V$.

To prove (ii), let $X$ be the set of all $f \in \operatorname{Pol}_{1} \mathbf{T}$ such that $f(u)=0$. Then set $F(x)=\Sigma_{f \in X} f(x)$. We get that $F(x) \in \operatorname{Pol}_{1} \mathbf{T}$ and $F(u)=0$. By Theorem 2.3, the subdirectly irreducible $\mathbf{T}$ is term equivalent to a mode of the form $\mathbf{S}(U)$ where $\mathbf{S}$ satisfies $(a)-(c)$ and $U$ satisfies $(i)-(i v)$ of Example 1. Therefore, if $u<s$, then there is an $f \in X=U-\left\{i d_{T}\right\}$ such that $f(u)=0<f(s) \leq F(s)$. This proves that $F^{-1}(0)=\{0, u\}$. Now suppose that $F(t) \neq t$ for some $t$ with $u<t$. By the remarks following Theorem 2.3, the fact that 0 is in the range of $F$ implies that $F \in\left\{q(x) \in \mathrm{Pol}_{1} \mathbf{A} \mid 0 \in q(A)\right\}=U$ and the set $U$ is a set of decreasing joinendomorphisms of the underlying semilattice of $\mathbf{T}$. Hence, we have $F(t)<t$. From $F^{-1}(0)=\{0, u\}$ and $u<t$ we get that $u \leq F(t)<t$. $\mathbf{T}$ is subdirectly irreducible, so there is a $p \in \operatorname{Pol}_{1} \mathbf{T}$ such that $p(F(t))=0<p(t)$. Since $p$ and $F$ both have 0 in their range, they both belong to $U$ and therefore they commute with each other. So, for $s$ $=p(t)$ we have

$$
F(s)=F(p(t))=p(F(t))=0<p(t)=s .
$$

Hence $s=u$. This gives us that

$$
p(p(t))=p(s)=p(u) \leq p(F(t))=0<u=s=p(t) .
$$

But this is impossible, as we explained in the first paragraph of this proof: since $p$ has 0 in its range, $p(p(t))=p(t)$. This contradiction proves that $F(t)=t$ for all $t$ with $u<t$. Choose $c(x, y)$ such that $c^{\mathbf{T}}(x, 0)=F(x)$ (which we can do since $F \in U$ ). Then $c^{\mathbf{W}}(u, 0)=0$ and $c^{\mathbf{T}}(t, 0)=t$ for $u<t$, so $c^{\mathbf{V}}\left(x, 0^{\mathbf{V}}\right)=x$ for all $x \in V$. This proves (ii).

To finish the first part of the lemma we must prove $(i i i)$. For this it suffices to show that if $u<r<s$ in $\mathbf{T}$, then there is a binary term $b(x, y)$ such that $b^{\mathbf{T}}(r, 0)=0<$ $u<b^{\mathbf{T}}(s, 0)$. This can be proved in essentially the same way that we proved (ii) with some simplifications. The sketch of the argument is as follows: Since $\mathbf{T}$ is subdirectly irreducible, there is a unary polynomial $F$ such that $F(r)=0<F(s)$. If $u<F(s)$, then choose any term $b(x, y)$ such that $b^{\mathbf{T}}(x, 0)=F(x)$ and we are done. Otherwise, $F(s)=u$ for every $F$ such that $F(r)=0$ and this forces

$$
F(F(s))=F(u) \leq F(r)=0<F(s) .
$$

But this case cannot occur, since then $F(x)$ would be a unary polynomial which is not idempotent, but has 0 in its range. This finishes the proof of the first part of the lemma.

Now assume that $\mathbf{V}$ and $\mathbf{W}$ are as described in the second statement of the lemma. Let $\mathbf{T}=(\mathbf{V} \times \mathbf{W}) / \theta$ where $\theta$ is the congruence on $\mathbf{V} \times \mathbf{W}$ generated by

$$
G=\left\{\left\langle\left(c, 0^{\mathbf{W}}\right),\left(c, u^{\mathbf{W}}\right)\right\rangle \mid c \in V-\left\{0^{\mathbf{V}}\right\}\right\} .
$$

If $\eta_{i}, i=0$ or 1 , is the kernel of the projection onto the $i^{\text {th }}$ coordinate of $\mathbf{V} \times \mathbf{W}$, then $\theta \leq \eta_{0}$ since $G \subseteq \eta_{0}$. To see that $\theta<\eta_{0}$, we must show that $\left\langle\left(0^{\mathbf{V}}, 0^{\mathbf{W}}\right),\left(0^{\mathbf{V}}, u^{\mathbf{W}}\right)\right\rangle \notin \theta$. Assume otherwise. Then we can find $\langle a, b\rangle \in G$ and a polynomial $p \in \operatorname{Pol}_{1} \mathbf{V} \times \mathbf{W}$ such that $p(a)=\left(0^{\mathbf{V}}, 0^{\mathbf{W}}\right) \neq p(b)$. We have $\langle a, b\rangle \in \eta_{0}$ and that each $\eta_{0^{-}}$class contains exactly 2 elements, so we get that $p(a)=\left(0^{\mathbf{V}}, 0^{\mathbf{W}}\right), p(b)=\left(0^{\mathbf{V}}, u^{\mathbf{W}}\right)$. Furthermore, 
$\{a, b\}=\left\{\left(c, 0^{\mathbf{W}}\right),\left(c, u^{\mathbf{W}}\right)\right\}$ for some $c \in V-\left\{0^{\mathbf{V}}\right\}$ since $\langle a, b\rangle \in G$. The element $\left(0 \mathbf{V}, 0^{\mathbf{W}}\right)$ is the least element of $\mathbf{V} \times \mathbf{W}$ and this element is in the range of polynomial $p(x)$. Therefore we can apply the equivalence of the conditions enumerated directly after the statement of Theorem 2.3 to obtain that $p(x)=b^{\mathbf{V} \times \mathbf{W}}\left(x,\left(0^{\mathbf{V}}, 0^{\mathbf{W}}\right)\right)$ for some binary term $b$. Altogether, this means that $b^{\mathbf{W}}\left(\left\{0^{\mathbf{W}}, u^{\mathbf{W}}\right\}, 0^{\mathbf{W}}\right)=\left\{0^{\mathbf{W}}, u^{\mathbf{W}}\right\}$ and $b^{\mathbf{V}}\left(c, 0^{\mathbf{V}}\right)=0^{\mathbf{V}}$. But $b^{\mathbf{W}}\left(x, 0^{\mathbf{W}}\right)$ is order-preserving in its first variable, so $b^{\mathbf{W}}\left(u^{\mathbf{W}}, 0^{\mathbf{W}}\right)$ $=u^{\mathbf{W}}$ while $b^{\mathbf{V}}\left(c, 0^{\mathbf{V}}\right)=0^{\mathbf{V}}$. Since $c \neq 0$, we have contradicted condition $(i)$. This contradiction shows that $\theta<\eta_{0}$, as we hoped.

To finish the proof of the second claim of the lemma we must show that $\mathbf{T}$ is subdirectly irreducible. Since $\left(0^{\mathbf{V}}, 0^{\mathbf{W}}\right)$ is the least element of $\mathbf{V} \times \mathbf{W},\left(0^{\mathbf{V}}, 0^{\mathbf{W}}\right) / \theta$ is the least element of $\mathbf{T}=(\mathbf{V} \times \mathbf{W}) / \theta$. If $(c, d) / \theta \in T$ and $c \neq 0 \mathbf{V}$, then since $\langle(c, d),(c, u)\rangle \in \theta$ we get $(c, d) / \theta=\left(c, u^{\mathbf{W}}\right) / \theta \geq\left(0^{\mathbf{V}}, u^{\mathbf{W}}\right) / \theta$. Therefore the element $\left(0^{\mathbf{V}}, u^{\mathbf{W}}\right) / \theta$ is the second least element of $\mathbf{T}$. We write $u^{\mathbf{T}}$ for $\left(0^{\mathbf{V}}, u^{\mathbf{W}}\right) / \theta$. To show that $\mathbf{T}$ is subdirectly irreducible, we need to show that for every $s<t$ in $\mathbf{T}$ there is a unary polynomial $p \in \mathrm{Pol}_{1} \mathbf{T}$ such that $p(s)=0^{\mathbf{T}}<p(t)$. If $s=0^{\mathbf{T}}$, then $p(x)=x$ works, so we may assume that $0<s<t$ and in particular that $u^{\mathbf{T}}<t$. We may assume therefore that $t=\left(f, u^{\mathbf{W}}\right) / \theta$ and $s=\left(e, u^{\mathbf{W}}\right) / \theta$ with $e<f$ in $\mathbf{V}$. To finish, it will suffice to show that there are polynomials $q(x), r(x) \in \operatorname{Pol}_{1} \mathbf{T}$ such that $r(s) \leq u^{\mathbf{T}}<r(t)$ and $q(x)=x$ for $x \neq u^{\mathbf{T}}$ while $q\left(u^{\mathbf{T}}\right)=0^{\mathbf{T}}$. For then $p(x)=q(r(x))$ is a polynomial where $p(s)=0^{\mathbf{T}}<p(t)$.

Using condition (iii) of the lemma and the fact that $e<f$ in $\mathbf{V}$, we can choose $b(x, y)$ such that $b^{\mathbf{V}}(e, 0)=0<b^{\mathbf{V}}(f, 0)$. Hence,

$$
b^{\mathbf{T}}\left(s, 0^{\mathbf{T}}\right) \leq u^{\mathbf{T}}<b^{\mathbf{T}}\left(t, 0^{\mathbf{T}}\right)
$$

and we may take $r(x)=b^{\mathbf{T}}\left(x, 0^{\mathbf{T}}\right)$. Now by condition $(i i)$ of the lemma there is a term $c(x, y)$ such that

$$
c^{\mathbf{W}}(u, 0)=0 \quad \text { and } \quad \forall x \in V\left(c^{\mathbf{V}}\left(x, 0^{\mathbf{V}}\right)=x\right) .
$$

Hence, for $q(x)=c^{\mathbf{T}}\left(x, 0^{\mathbf{T}}\right)$ we have $q\left(u^{\mathbf{T}}\right)=0^{\mathbf{T}}$ and $q(x)=x$ for $x \neq u^{\mathbf{T}}$. This finishes the proof that $\mathbf{T}$ is subdirectly irreducible.

What remains is to show that under the conditions of the third part of the lemma the function

$$
f: \mathbf{T}^{\prime} \rightarrow \mathbf{T}:(v, w) / \theta \mapsto \begin{cases}v & \text { if } v>0 \\ w & \text { if } v=0\end{cases}
$$

is an isomorphism. Equivalently, we must show that

$$
g: \mathbf{V} \times \mathbf{W} \rightarrow \mathbf{T}:(v, w) \mapsto \begin{cases}v & \text { if } v>0 \\ w & \text { if } v=0\end{cases}
$$

is a surjective homomorphism with kernel $\theta$. The only nontrivial part of this verification is that $g$ is a homomorphism. We proceed with that verification. (Be alert to the fact that $0^{\mathbf{W}}=0^{\mathbf{T}}=0$, but $0^{\mathbf{V}}=u^{\mathbf{T}}$.)

There is no loss in generality if we assume that $\mathcal{V}=\mathrm{V}(\mathbf{T})$ and by making this assumption we get (by Theorem 2.3) that $\mathcal{V}$ is equivalent to a variety where all the basic operations are binary. So it suffices to show that if $b(x, y)$ is a binary term, then

$$
g\left(b^{\mathbf{V} \times \mathbf{W}}\left((v, w),\left(v^{\prime}, w^{\prime}\right)\right)\right)=b^{\mathbf{T}}\left(g((v, w)), g\left(\left(v^{\prime}, w^{\prime}\right)\right)\right) .
$$


By symmetry, we need to consider only the cases: (1) $v=0^{\mathbf{V}}=v^{\prime},(2) v \neq 0^{\mathbf{V}}=v^{\prime}$ and (3) $v \neq 0^{\mathbf{V}} \neq v^{\prime}$. For Case (1) we simply have

$$
\begin{aligned}
g\left(b^{\mathbf{V} \times \mathbf{W}}\left(\left(0^{\mathbf{V}}, w\right),\left(0^{\mathbf{V}}, w^{\prime}\right)\right)\right) & =g\left(0^{\mathbf{V}}, b^{\mathbf{W}}\left(w, w^{\prime}\right)\right) \\
& =b^{\mathbf{W}}\left(w, w^{\prime}\right) \\
& =b\left(g\left(\left(0^{\mathbf{V}}, w\right)\right), g\left(\left(0^{\mathbf{V}}, w^{\prime}\right)\right)\right) .
\end{aligned}
$$

For Case (2) we must show that

$$
\begin{aligned}
g\left(b^{\mathbf{V} \times \mathbf{W}}\left((v, w),\left(0^{\mathbf{V}}, w^{\prime}\right)\right)\right) & =g\left(b^{\mathbf{V}}\left(v, 0^{\mathbf{V}}\right), b^{\mathbf{W}}\left(w, w^{\prime}\right)\right) \\
& =b^{\mathbf{T}}\left(v, w^{\prime}\right) \\
& =b^{\mathbf{T}}(v, 0)+b^{\mathbf{T}}\left(0, w^{\prime}\right) .
\end{aligned}
$$

If $b^{\mathbf{V}}\left(v, 0^{\mathbf{V}}\right)>0^{\mathbf{V}}$, then we have

$$
g\left(b^{\mathbf{V}}\left(v, 0^{\mathbf{V}}\right), b^{\mathbf{W}}\left(w, w^{\prime}\right)\right)=b^{\mathbf{V}}\left(v, 0^{\mathbf{V}}\right)=b^{\mathbf{T}}(v, 0)>u .
$$

But $b^{\mathbf{T}}\left(0, w^{\prime}\right)=b^{\mathbf{W}}\left(0, w^{\prime}\right) \in\{0, u\}$, so in this case

$$
g\left(b^{\mathbf{V}}\left(v, 0^{\mathbf{V}}\right), b^{\mathbf{W}}\left(w, w^{\prime}\right)\right)=b^{\mathbf{T}}(v, 0)=b^{\mathbf{T}}(v, 0)+b^{\mathbf{T}}\left(0, w^{\prime}\right)=b^{\mathbf{T}}\left(v, w^{\prime}\right)
$$

just as we hoped. Now suppose that $b^{\mathbf{V}}\left(v, 0^{\mathbf{V}}\right)=0^{\mathbf{V}}$. To settle Case (2) we must verify the third and fifth equalities in

$$
\begin{aligned}
g\left(b^{\mathbf{V}}\left(v, 0^{\mathbf{V}}\right), b^{\mathbf{W}}\left(w, w^{\prime}\right)\right) & =b^{\mathbf{W}}\left(w, w^{\prime}\right) \\
& =b^{\mathbf{W}}(w, 0)+b^{\mathbf{W}}\left(0, w^{\prime}\right) \\
& =b^{\mathbf{W}}\left(0, w^{\prime}\right) \\
& =b^{\mathbf{T}}\left(0, w^{\prime}\right) \\
& =b^{\mathbf{T}}(v, 0)+b^{\mathbf{T}}\left(0, w^{\prime}\right) \\
& =b^{\mathbf{T}}\left(v, w^{\prime}\right) .
\end{aligned}
$$

The only way the third equality could fail is for $b^{\mathbf{W}}\left(0, w^{\prime}\right)=0$ and for $b^{\mathbf{W}}(w, 0)=u$. In this case we must have $w=u$ and so $b^{\mathbf{W}}(u, 0)=u$. But we have $b^{\mathbf{V}}(v, 0)=0$ and $v \neq 0$. This is a contradiction to property $(i)$ of the lemma. If the fifth equality fails, then we must have $b^{\mathbf{W}}\left(0, w^{\prime}\right)=0$ and

$$
0<b^{\mathbf{T}}(v, 0) \leq b^{\mathbf{T}}(v, 0)+u=b^{\mathbf{V}}\left(v, 0^{\mathbf{V}}\right)=0^{\mathbf{V}}=u .
$$

Hence, $b^{\mathbf{T}}(v, 0)=u$. But $b^{\mathbf{V}}\left(v, 0^{\mathbf{V}}\right)=0^{\mathbf{V}}$ and $v>0 \mathbf{V}$, so property $(i)$ shows that $0=$ $b^{\mathbf{W}}(u, 0)=b^{\mathbf{T}}(u, 0)$. We conclude that

$$
b^{\mathbf{T}}\left(b^{\mathbf{T}}(v, 0), 0\right)=0<u=b^{\mathbf{T}}(v, 0) .
$$

We have produced a unary polynomial $b^{\mathbf{T}}(x, 0)$ which is not idempotent but has 0 in its range. This is impossible, as we observed at the beginning of the proof. Hence, the proof in Case (2) is complete. Finally, assume that we are in Case (3). We must show that

$$
g\left(b^{\mathbf{V} \times \mathbf{W}}\left((v, w),\left(v^{\prime}, w^{\prime}\right)\right)\right)=b^{\mathbf{T}}\left(v, v^{\prime}\right) .
$$

Now $b^{\mathbf{V} \times \mathbf{W}}\left((v, w),\left(v^{\prime}, w^{\prime}\right)\right)=\left(b^{\mathbf{V}}\left(v, v^{\prime}\right), b^{\mathbf{W}}\left(w, w^{\prime}\right)\right)$, so if $b^{\mathbf{V}}\left(v, v^{\prime}\right) \neq 0$, then both sides of the last displayed equation are equal to $b^{\mathbf{T}}\left(v, v^{\prime}\right)$. Suppose now that $b^{\mathbf{V}}\left(v, v^{\prime}\right)=$ $0^{\mathbf{V}}$. We then get $0^{\mathbf{V}}=b^{\mathbf{V}}\left(v, v^{\prime}\right)=b^{\mathbf{V}}\left(v, 0^{\mathbf{V}}\right)+b^{\mathbf{V}}\left(0^{\mathbf{V}}, v^{\prime}\right)$. Then $b^{\mathbf{V}}\left(v, 0^{\mathbf{V}}\right)=0^{\mathbf{V}}$, 
$v>0 \mathbf{V}$, and $b^{\mathbf{V}}\left(0^{\mathbf{V}}, v^{\prime}\right)=0^{\mathbf{V}}, v^{\prime}>0^{\mathbf{V}}$. By property $(i)$ of the lemma, these conditions imply $b^{\mathbf{W}}(w, 0)=0$ and $b^{\mathbf{W}}\left(0, w^{\prime}\right)=0$ and consequently that $b^{\mathbf{W}}\left(w, w^{\prime}\right)=b^{\mathbf{W}}(w, 0)+$ $b^{\mathbf{W}}\left(0, w^{\prime}\right)=0$. So when we are in the situation that $b^{\mathbf{V}}\left(v, v^{\prime}\right)=0^{\mathbf{V}}$ holds in Case (3), then we get that $b^{\mathbf{W}}\left(w, w^{\prime}\right)=0$ and $g\left(b^{\mathbf{V} \times \mathbf{W}}\left((v, w),\left(v^{\prime}, w^{\prime}\right)\right)\right)=0$. We need to show that $0=b^{\mathbf{T}}\left(v, v^{\prime}\right)=b^{\mathbf{T}}(v, 0)+b^{\mathbf{T}}\left(0, v^{\prime}\right)$. By symmetry, it is enough to show that $b^{\mathbf{T}}(v, 0)=0$. Now, we know that in $\mathbf{V}$ we have $b^{\mathbf{V}}\left(v, 0^{\mathbf{V}}\right)=0^{\mathbf{V}}$ and the element $0^{\mathbf{V}} \in V$ is none other than the element $u \in W \subseteq T$. We also have $b^{\mathbf{W}}(u, 0)=0$. Together this gives us that

$$
b^{\mathbf{T}}(v, 0) \leq u=b^{\mathbf{V}}\left(v, 0^{\mathbf{V}}\right)
$$

and

$$
b^{\mathbf{T}}(u, 0)=b^{\mathbf{W}}(u, 0)=0 .
$$

Hence, $b^{\mathbf{T}}\left(b^{\mathbf{T}}(v, 0), 0\right)=0$. Since unary polynomials which have 0 in their range are idempotent, $b^{\mathbf{T}}(v, 0)=0$ as desired. This completes the proof of the lemma.

LEMMA 3.6 Assume that $\mathcal{V}$ is a variety of semilattice modes and $\mathbf{R}(\mathcal{V})$ is a finite, bounded distributive lattice. If $\left(\mathbf{A} ; f_{1}, \mathbf{B} ; g_{1}, \mathbf{C}\right)$ is an amalgam where

(i) $f_{1}$ and $g_{1}$ are inclusion homomorphisms,

(ii) $\mathbf{B}$ is subdirectly irreducible,

(iii) $\mathbf{C}$ is an essential extension of $\mathbf{A}$ in $\mathcal{V}$,

(iv) $A=B-\left\{0^{\mathbf{B}}\right\}$,

then it is possible to complete $\left(\mathbf{A} ; f_{1}, \mathbf{B} ; g_{1}, \mathbf{C}\right)$ in $\mathcal{V}$.

Proof: The function $f: \mathbf{B} \rightarrow \mathbf{B}: x \mapsto x+u$, where $u$ is the least element of $\mathbf{B}-\left\{0^{\mathbf{B}}\right\}$, is an endomorphism of $\mathbf{B}$ with kernel $\mu$ and image $A$. Hence, the induced function $\bar{f}: \mathbf{B} / \mu \rightarrow \mathbf{A}$ is an isomorphism. Let $\mathbf{W}=\operatorname{Sg}^{\mathbf{B}}(\{0, u\})$. Then $\mathbf{A}$ and $\mathbf{W}$ are related by conditions $(i)-($ iii) of Lemma 3.5. Our goal will be to show that since $\mathbf{C}$ is an essential extension of $\mathbf{A}$, then $\mathbf{C}$ and $\mathbf{W}$ are also related by conditions $(i)-(i i i)$ of Lemma 3.5. Hence, there is a subdirectly irreducible $\mathbf{D} \in \mathcal{V}$ constructed as in the proof of Lemma 3.5. We then verify that $\mathbf{D}$ together with obvious maps of $\mathbf{B}$ and $\mathbf{C}$ into $\mathbf{D}$ completes $\left(\mathbf{A} ; f_{1}, \mathbf{B} ; g_{1}, \mathbf{C}\right)$ in $\mathcal{V}$. Before proceeding with the argument, we point out that each of $\mathbf{A}, \mathbf{B}$ and $\mathbf{C}$ is finite. $\mathbf{B}$ is finite because of Corollary 2.5 and the fact that $\mathbf{R}(\mathcal{V})$ is finite. $\mathbf{A}$ is a subalgebra of $\mathbf{B}$, so $\mathbf{A}$ is finite. It is proven in [2] that when $\mathcal{V}$ is residually small and has the congruence extension property, then any essential extension of a finite algebra is finite. Hence $\mathbf{C}$ is finite.

Our first step will be to show that $\mathbf{C}$ and $\mathbf{W}$ are related by conditions $(i)-($ iii $)$ of Lemma 3.5 ( $\mathbf{C}$ is playing the role of $\mathbf{V}$ in Lemma 3.5). Assume that Lemma $3.5(i)$ fails to hold for $\mathbf{C}$ and $\mathbf{W}$. Then there is a binary term $b(x, y)$ such that $b^{\mathbf{W}}(u, 0)=u$, but $p(x)=b^{\mathbf{C}}\left(x, 0^{\mathbf{C}}\right)$ is not the identity function. $p(x)$ is a decreasing endomorphism, so since $\mathbf{C}$ is finite we get that $\operatorname{ker} p$ is nontrivial. $\mathbf{A}$ is essentially embedded in $\mathbf{C}$, so $\left.($ ker $p)\right|_{A}>00_{A}$. But by Lemma 3.2 we get that $g_{1}\left(0^{\mathbf{A}}\right)=0^{\mathbf{C}}$. In fact, $0^{\mathbf{A}}=0^{\mathbf{C}}$ since $g_{1}$ is inclusion. Thus, $p(x) \in \operatorname{Pol}_{1} \mathbf{A}$ and ker $p>0_{A}$. But now $b^{\mathbf{W}}(u, 0)=u$ and $p(x)$ $=b^{\mathbf{A}}\left(x, 0^{\mathbf{A}}\right)=\left.b^{\mathbf{C}}\left(x, 0^{\mathbf{C}}\right)\right|_{A}$ is not the identity function on $A$. This is impossible since $\mathbf{A}$ and $\mathbf{W}$ ARE related as in Lemma 3.5. Now we show that $\mathbf{C}$ and $\mathbf{W}$ are related as in condition (ii). Since $\mathbf{A}$ and $\mathbf{W}$ are related as in condition $(i i)$, there a term $c(x, y)$ 
such that $c^{\mathbf{W}}(u, 0)=0$ and $c^{\mathbf{A}}\left(x, 0^{\mathbf{A}}\right)$ is the identity function on $A$. By the same reasoning we used for part $(i), c^{\mathbf{C}}\left(x, 0^{\mathbf{C}}\right)$ is the identity function on $C$. Now assume that condition (iii) fails for $\mathbf{C}$ and $\mathbf{W}$. Then there are elements $e<f$ and whenever $b(x, y)$ is a binary term we have

$$
b^{\mathbf{C}}\left(e, 0^{\mathbf{C}}\right)=0 \leftrightarrow b^{\mathbf{C}}\left(f, 0^{\mathbf{C}}\right)=0 .
$$

But, the conditions enumerated after the statement of Theorem 2.3 show that any unary polynomial of $\mathbf{C}$ which has 0 in its range is of the form $b^{\mathbf{C}}\left(x, 0^{\mathbf{C}}\right)$ for some binary term. Hence, we have that for any $p(x) \in \mathrm{Pol}_{1} \mathbf{C}$

$$
p(e)=0 \leftrightarrow p(f)=0 .
$$

Thus, the $\operatorname{Cg}(e, f)$-class containing $0^{\mathbf{C}}$ is just $\left\{0^{\mathbf{C}}\right\}$. Let $\theta=\left.\operatorname{Cg}(e, f)\right|_{\mathbf{A}}$. Since $0^{\mathbf{A}}=0^{\mathbf{C}}$ we get

$$
\left\{0^{\mathbf{A}}\right\} \subseteq 0^{\mathbf{A}} / \theta \subseteq 0^{\mathbf{C}} / \mathrm{Cg}(e, f)=\left\{0^{\mathbf{C}}\right\}
$$

and so $0^{\mathbf{A}} / \theta=\left\{0^{\mathbf{A}}\right\}$. But $\operatorname{Cg}(e, f)$ is not the trivial congruence on $\mathbf{C}, \operatorname{so} \theta=\left.\operatorname{Cg}(e, f)\right|_{\mathbf{A}}$ is not the trivial congruence on $\mathbf{A}$. Choose $\left(e^{\prime}, f^{\prime}\right) \in \theta$ with $e^{\prime}<f^{\prime}$. Since $\mathbf{A}$ and $\mathbf{W}$ are related as in condition (iii), there is a term $b(x, y)$ such that $b^{\mathbf{A}}\left(e^{\prime}, 0^{\mathbf{A}}\right)=0^{\mathbf{A}}<$ $b^{\mathbf{A}}\left(f^{\prime}, 0^{\mathbf{A}}\right)$. This contradicts the fact that the $\theta$-class of $0^{\mathbf{A}}$ is just $\left\{0^{\mathbf{A}}\right\}$. With this we complete the proof that $\mathbf{C}$ and $\mathbf{W}$ are related in Lemma 3.5.

Now let $\theta$ be the congruence on $\mathbf{C} \times \mathbf{W}$ generated by

$$
G=\left\{\left\langle\left(c, 0^{\mathbf{W}}\right),\left(c, u^{\mathbf{W}}\right)\right\rangle \mid c \in C-\left\{0^{\mathbf{C}}\right\}\right\} .
$$

Lemma 3.5 proves that $\mathbf{D}=(\mathbf{C} \times \mathbf{W}) / \theta$ is subdirectly irreducible. The congruence $\theta^{\prime}$ $=\left.\theta\right|_{\mathbf{A} \times \mathbf{W}}$ is just the congruence generated by

$$
G=\left\{\left\langle\left(a, 0^{\mathbf{W}}\right),\left(a, u^{\mathbf{W}}\right)\right\rangle \mid a \in A-\left\{0^{\mathbf{A}}\right\}\right\} .
$$

Thus, $(\mathbf{A} \times \mathbf{W}) / \theta^{\prime}$ is subdirectly irreducible and, by the third part of Lemma 3.5, isomorphic to $\mathbf{B}$. Define $f_{2}: \mathbf{B} \rightarrow \mathbf{D}$ be the function

$$
f_{2}(b)= \begin{cases}\left(b, u^{\mathbf{W}}\right) / \theta & \text { if } b \in A \\ \left(0^{\mathbf{C}}, 0^{\mathbf{W}}\right) / \theta & \text { if } b \notin A\end{cases}
$$

Then $f_{2}$ is just an isomorphism of $\mathbf{B}$ onto $(\mathbf{A} \times \mathbf{W}) / \theta^{\prime}$ which carries $A$ onto $(A \times$ $\left.\left\{u^{\mathbf{W}}\right\}\right) / \theta^{\prime}$ in the natural way. Now define $g_{2}: \mathbf{C} \rightarrow \mathbf{D}$ by $g_{2}(c)=\left(c, u^{\mathbf{W}}\right) / \theta$. $g_{2}$ is an isomorphism of $\mathbf{C}$ onto the subalgebra of $\mathbf{D}$ of elements $>\left(0^{\mathbf{C}}, 0^{\mathbf{W}}\right) / \theta \cdot g_{2}$ also carries $A$ onto $\left(A \times\left\{u^{\mathbf{W}}\right\}\right) / \theta^{\prime}$ in the natural way. Thus, $f_{2}$ and $g_{2}$ are embeddings and $f_{2} \circ f_{1}$ $=g_{2} \circ g_{1}$. This proves that $\left(f_{2} ; g_{2} ; \mathbf{D}\right)$ completes $\left(\mathbf{A} ; f_{1}, \mathbf{B} ; g_{1}, \mathbf{C}\right)$.

THEOREM 3.7 If $\mathcal{V}$ is a locally finite variety of semilattice modes where $\mathbf{R}(\mathcal{V})$ is a bounded distributive lattice, then $\mathcal{V}$ has the AP.

Proof: We need to show that we can complete every amalgam $\left(\mathbf{A} ; f_{1}, \mathbf{B} ; g_{1}, \mathbf{C}\right)$ where $|A|>1$, B is subdirectly irreducible and $g_{1}$ is essential. Assume instead that $\left(\mathbf{A} ; f_{1}, \mathbf{B} ; g_{1}, \mathbf{C}\right)$ cannot be completed in $\mathcal{V}$ and that $k$ is the height of some element $f_{1}\left(a_{0}\right) \in B, a_{0} \in A$, which has minimum height among all elements of the form $f_{1}(a)$, 
$a \in A$. We may assume that our triple is chosen so that the value $k$ is minimal for all triples $\left(\mathbf{A}^{\prime} ; f_{1}^{\prime}, \mathbf{B}^{\prime} ; g_{1}^{\prime}, \mathbf{C}^{\prime}\right)$ of the form above which cannot be completed in $\mathcal{V}$.

Case 1. $k=0$.

In this case $f_{1}\left(a_{0}\right)=0^{\mathbf{B}}$, so necessarily $a_{0}$ is the least element of $\mathbf{A}$ : i.e., $a_{0}=0^{\mathbf{A}}$. By Lemma 3.2 we get that $g_{1}\left(0^{\mathbf{A}}\right)=0^{\mathbf{C}}$. So Lemma 3.3 now proves that this case can not occur.

Case 2. $k>0$.

In this case $u^{\mathbf{B}} \leq f_{1}(a)$ for all $a \in A$. Let $\mathbf{B}^{\prime} \leq \mathbf{B}$ be the subalgebra of all elements of $\mathbf{B}$ which are $\geq u^{\mathbf{B}}$. Thus, $\mathbf{B}^{\prime}$ is the image of the endomorphism $\mathbf{B} \rightarrow \mathbf{B}: x \mapsto x+u^{\mathbf{B}}$. The triple $\left(\mathbf{A} ; f_{1}, \mathbf{B}^{\prime} ; g_{1}, \mathbf{C}\right)$ can be completed in $\mathcal{V}$ by induction. The argument which proves this is the same as the argument used in the proof of Lemma 3.1. One simply takes a subdirect representation of $\mathbf{B}^{\prime}$, obtains a representation of $\mathbf{A}$ by restriction and then uses the congruence extension property to extend the representation in a maximal way to $\mathbf{C}$. This gives a family of amalgams of the form $\left(\mathbf{A} / \theta_{i}^{\mathbf{A}} ; f_{1}^{(i)}, \mathbf{B}^{\prime} / \theta_{i} ; g_{1}^{(i)}, \mathbf{C} / \theta_{i}^{\mathbf{C}}\right)$ where each $\mathbf{B}^{\prime} / \theta_{i}$ is a subdirectly irreducible homomorphic image of $\mathbf{B}^{\prime}$ and each $g_{1}^{(i)}$ is essential. If all can be completed, then a product completes the original amalgam. But, by induction, they all can be completed. Each $\mathbf{A} / \theta_{i}^{\mathbf{A}}$ contains $\bar{a}_{0}=a_{0} / \theta_{i}^{\mathbf{A}}$ and the height of $f_{1}^{(i)}\left(\bar{a}_{0}\right)$ in $\mathbf{B}^{\prime} / \theta_{i}$ is no more than the height of $f_{1}\left(a_{0}\right)$ in $\mathbf{B}^{\prime}$. But this latter height is at most $k-1$ since $\mathbf{B}^{\prime}$ was obtained from $\mathbf{B}$ by removing the bottom element. Thus we get that $\left(\mathbf{A} ; f_{1}, \mathbf{B}^{\prime} ; g_{1}, \mathbf{C}\right)$ can be completed, as claimed. Let $\left(f_{2} ; g_{2} ; \mathbf{D}\right)$ be a triple which completes it. We may assume that $f_{2}$ is essential (since $g_{1}$ was) and we also may assume that $f_{2}$ is inclusion.

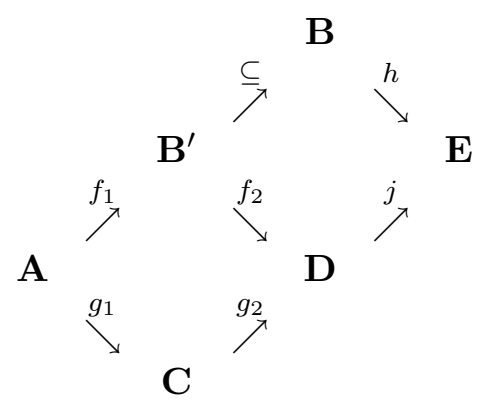

Now Lemma 3.6 proves that $\left(\mathbf{B}^{\prime} ; \subseteq, \mathbf{B} ; f_{2}, \mathbf{D}\right)$ may be completed, say by $(h ; j ; \mathbf{E})$. Then $\left(h ; j \circ g_{2} ; \mathbf{E}\right)$ completes our original amalgam $\left(\mathbf{A} ; f_{1}, \mathbf{B} ; g_{1}, \mathbf{C}\right)$. This contradicts our assumption that $\left(\mathbf{A} ; f_{1}, \mathbf{B} ; g_{1}, \mathbf{C}\right)$ cannot be completed in $\mathcal{V}$ and completes the proof of the theorem.

THEOREM 3.8 If $\mathcal{V}$ is a variety of semilattice modes where $\mathbf{R}(\mathcal{V})$ is a bounded distributive lattice, then $\mathcal{V}$ has the AP.

Proof: For the purpose of obtaining a contradiction, assume that $\mathcal{V}$ is a variety of semilattice modes where $\mathbf{R}(\mathcal{V})$ is a bounded distributive lattice, but $\mathcal{V}$ does not have the AP. Then there is an amalgam $\left(\mathbf{A} ; f_{1}, \mathbf{B} ; g_{1}, \mathbf{C}\right)$ which cannot be completed in $\mathcal{V}$. We shall show how to obtain from $\mathcal{V}$ a variety $\mathcal{U}$ with all of these properties, but also the property that $\mathcal{U}$ is of finite similarity type. Then, by Theorem $2.2, \mathbf{R}(\mathcal{U})$ 
is a finitely generated, bounded distributive lattice. Bounded distributive lattices are locally finite, so in fact $\mathbf{R}(\mathcal{U})$ is finite. But by Corollary 2.5 this implies that $\mathcal{U}$ has only finitely many subdirectly irreducible members and all are finite. It follows that $\mathcal{U}$ is a locally finite variety. Now Theorem 3.7 proves the nonexistence of such a $\mathcal{U}$ and therefore the nonexistence of $\mathcal{V}$.

If $\mathbf{D}$ is an algebra, let $\mathbf{D}_{D}$ be the expansion of $\mathbf{D}$ obtained by letting each element of $\mathbf{D}$ be the interpretation of a new constant symbol. The diagram of $\mathbf{D}$ is the collection $\Delta_{\mathbf{D}}$ of all atomic and negations of atomic sentences which hold in $\mathbf{D}_{D}$. If $\Sigma$ is a set of sentences in the language of $\mathbf{D}$, then $\Sigma \cup \Delta_{\mathbf{D}}$ has a model iff $\Sigma$ has a model $\mathbf{E}$ which has a submodel isomorphic to $\mathbf{D}$. This claim is Proposition 2.1.8 of [1].

Now, recall that $\left(\mathbf{A} ; f_{1}, \mathbf{B} ; g_{1}, \mathbf{C}\right)$ cannot be completed in $\mathcal{V}$. In this paragraph we shall expand the language $\mathcal{L}$ of $\mathcal{V}$ by adding new constant symbols $\left\{k_{b} \mid b \in B\right\}$ for elements of $B$ and new constant symbols $\left\{k_{c} \mid c \in C\right\}$ for elements of $C$. We assume that all new symbols are distinct from the symbols of $\mathcal{L}$ and distinct from each other. Let $\Delta_{\mathbf{B}}$ be the diagram of $\mathbf{B}$, let $\Delta_{\mathbf{C}}$ be the diagram of $\mathbf{C}$ and let $\Sigma$ be the set of equations holding in $\mathcal{V}$. Let $\Upsilon$ be the set of sentences of the form $k_{b}=k_{c}$ where there is some $a \in A$ such that $f_{1}(a)=b, g_{1}(a)=c$. By a trivial modification of the argument in [1] the set of sentences $\Sigma \cup \Delta_{\mathbf{B}} \cup \Delta_{\mathbf{C}} \cup \Upsilon$ has a model iff $\Sigma$ has a model containing submodels $\mathbf{A}^{\prime} \cong_{h} \mathbf{A}, \mathbf{B}^{\prime} \cong_{j} \mathbf{B}$ and $\mathbf{C}^{\prime} \cong_{k} \mathbf{C}$ where $j^{-1} \circ f_{1} \circ h=\subseteq$ and $k^{-1} \circ g_{1} \circ h$ $=\subseteq$. (Here $\mathbf{A}^{\prime} \cong_{h} \mathbf{A}$ indicates that $h$ is an isomorphism from $\mathbf{A}^{\prime}$ onto $\mathbf{A}$.) In other words, $\Sigma \cup \Delta_{\mathbf{B}} \cup \Delta_{\mathbf{C}} \cup \Upsilon$ has a model iff $\left(\mathbf{A} ; f_{1}, \mathbf{B} ; g_{1}, \mathbf{C}\right)$ can be completed in $\mathcal{V}$. By assumption, we cannot complete this amalgam, so $\Sigma \cup \Delta_{\mathbf{B}} \cup \Delta_{\mathbf{C}} \cup \Upsilon$ has no model. But this means that there is a finite subset $\Sigma_{0}$ where $\Sigma_{0} \subseteq \Sigma \cup \Delta_{\mathbf{B}} \cup \Delta_{\mathbf{C}} \cup \Upsilon$ and $\Sigma_{0}$ has no model. Let $\sigma$ be a finite set of operation symbols of $\mathcal{V}$ which includes at least all of the operation symbols occurring in sentences of $\Sigma_{0}$ together with enough operation symbols to express the term $x+y$. Let $\Sigma^{\prime}$ be the set of all equations in $\Sigma$ that involve only the symbols in $\sigma$. We define $\mathcal{U}$ to be the variety of type $\sigma$ which is axiomatized by the equations in $\Sigma^{\prime} . \mathcal{U}$ is a variety of modes because the idempotent and entropic laws for the symbols in $\sigma$ are equations in $\Sigma$ involving only symbols in $\sigma$. Hence, these laws are equations of $\mathcal{U}$. There are equations in $\Sigma$ which involve only the symbols in $\sigma$ expressing the fact that $x+y$ is a semilattice operation. Hence, these are equations of $\mathcal{U}$. There are equations of $\Sigma$ expressing the fact that each coefficient of a term of type $\sigma$ is idempotent in $\mathbf{R}(\mathcal{V})$. These equations belong to $\Sigma^{\prime}$, so $\mathbf{R}(\mathcal{U})$ satisfies $x^{2}=x$ and must be a bounded distributive lattice. Finally, the reducts of $\mathbf{A}, \mathbf{B}$ and $\mathbf{C}$ to the symbols in $\sigma$ satisfy $\Sigma^{\prime}$ and therefore belong to $\mathcal{U}$. If we show that the amalgam of reducts $\left(\mathbf{A} ; f_{1}, \mathbf{B} ; g_{1}, \mathbf{C}\right)$ cannot be completed in $\mathcal{U}$, then we will have established that $\mathcal{U}$ has all the properties of $\mathcal{V}$, but has finite type as well. But clearly, if $\left(f_{2} ; g_{2} ; \mathbf{D}\right)$ completes this amalgam in $\mathcal{U}$, then we can construct from $\left(f_{2} ; g_{2} ; \mathbf{D}\right)$ a model of $\Sigma_{0}$ in the obvious way: the model is the expansion of $\mathbf{D}$ obtained by using $f_{2}$ and $g_{2}$ to interpret the constant symbols. Hence, the amalgam of reducts $\left(\mathbf{A} ; f_{1}, \mathbf{B} ; g_{1}, \mathbf{C}\right)$ cannot be completed in $\mathcal{U}$. $\square$.

We now begin the proof that if $\mathcal{V}$ is a locally finite variety of semilattice modes which has the AP, then $\mathbf{R}(\mathcal{V})$ is a bounded distributive lattice. We shall argue the contrapositive and the argument will proceed as follows. Assume that $\mathbf{R}(\mathcal{V})$ is not a bounded distributive lattice. Let $\mathcal{U} \subseteq \mathcal{V}$ be a subvariety of $\mathcal{V}$ which is minimal for the property that $\mathbf{R}(\mathcal{U})$ is not a bounded distributive lattice. (Such a $\mathcal{U}$ exists, since it is a consequence of Theorem 2.7 that a locally finite variety of semilattice modes has 
only finitely many subvarieties.) We shall show that $\mathcal{U}$ is contained in no locally finite variety of modes which has the AP. This will prove that $\mathcal{V}$ did not have the AP.

We will need to understand the structure of $\mathbf{R}(\mathcal{U})$. If $\mathcal{U}=\bigvee_{i \in I} \mathcal{U}_{i}$ where each $\mathcal{U}_{i}$ is a proper subvariety of $\mathcal{U}$, then $\mathbf{R}(\mathcal{U})$ is a proper subdirect product of the semirings $\mathbf{R}\left(\mathcal{U}_{i}\right)$ each of which is a bounded distributive lattice. This would imply that $\mathbf{R}(\mathcal{U})$ is a bounded distributive lattice which is false. We conclude that $\mathcal{U}$ is a join-irreducible subvariety of $\mathcal{V}$. Hence, by Theorem 2.7, 0 is a meet-irreducible congruence of the (finite) semiring $\mathbf{R}(\mathcal{U})$ and therefore $\mathbf{R}(\mathcal{U})$ is subdirectly irreducible. If $\mu$ is the monolith of $\mathbf{R}$, then $\mathbf{R}(\mathcal{U}) / \mu$ is a bounded distributive lattice. The next result describes the structure of $\mathbf{R}(\mathcal{U})$. In this section, we will call a semiring $\mathbf{R}$ minimal if it is finite, satisfies all the identities listed in Theorem 2.1, fails to be a bounded distributive lattice, but any proper homomorphic image is a bounded distributive lattice.

LEMMA 3.9 If $\mathbf{R}$ is minimal with monolith $\mu$, then the following are true:

(i) 0 is a meet-irreducible element of $\langle R ;+\rangle$ and 1 is a join-irreducible element of $\langle R ;+\rangle$.

(ii) $\langle R-\{0,1\} ;+\rangle \cong\langle\{0,1\},+\rangle^{n}$ for some $n$.

(iii) If $0^{\prime}$ is the least element in the semilattice order on $R-\{0\}$, then $\mu$ is the equivalence relation on $R$ generated by $\left(0,0^{\prime}\right)$.

(iv) If $r \in R-\{1\}$, then $r \cdot 0^{\prime}=0$.

Proof: First we show that the only nontrivial $\mu$-class is $0 / \mu$. Since $\mathbf{R} / \mu$ is a distributive lattice, we get that $\left(r, r^{2}\right) \in \mu$ for all $r \in R$. Since $\mathbf{R}$ is not a distributive lattice, there exists an element $r \in R$ such that $r \neq r^{2}$. (See the remarks following Theorem 2.1.) Then, since $|R|$ is finite and $r^{n}=r^{n}(1+r)=r^{n}+r^{n+1}$, we get that

$$
r>r^{2} \geq r^{3} \geq \cdots \geq r^{n}=e=e^{2}
$$

for some $n$. Let $\mathbf{S}$ be the semiring with universe $S=\{x \in R \mid x \geq e\}$ which is defined as $\langle S ; \cdot,+, 1, e\rangle$. We claim that the map $\phi: \mathbf{R} \rightarrow \mathbf{S}$ defined by $x \mapsto x+e$ is a surjective homomorphism for which $\phi(r)=r \neq r^{2}=\phi\left(r^{2}\right)$. The only non-obvious part of this claim is that $\phi$ preserves multiplication. This can be shown as follows:

$$
\begin{aligned}
\phi(x) \phi(y) & =(x+e)(y+e) \\
& =x y+e x+e y+e^{2} \\
& =x y+e x+e y+e \\
& =x y+e(1+(x+y)) \\
& =x y+e \\
& =\phi(x y) .
\end{aligned}
$$

Since $\left(r, r^{2}\right) \in \mu$ and $\left(r, r^{2}\right) \notin$ ker $\phi$, we conclude that $\phi$ is $1-1$ and therefore $e=0$. Hence, if $r \neq r^{2}$, there is an $n$ such that

$$
r \mu r^{2} \mu \cdots \mu r^{n}=0 .
$$

An element $r$ will be called nilpotent if there is an $n$ such that $r^{n}=0$. The set of nilpotent elements of $\mathbf{R}$ is clearly an annihilator ideal, I. Now assume that 
$a \in R-I$ and $r \in I$. Then $a+r \in R-I$ since $I$ is an order-ideal. Hence, $a^{2}=a$ and $(a+r)^{2}=a+r$. This gives us that

$$
\begin{aligned}
a+r & =(a+r)^{2} \\
& =a^{2}+a r+r^{2} \\
& =a+a r+r^{2} \\
& =a(1+r)+r^{2} \\
& =a+r^{2} \\
& =a+r^{4}=\cdots=a .
\end{aligned}
$$

Hence, every element not in $I$ is strictly above every element in $I$.

The ideal congruence $\Theta(I)=\left\{(x, y) \in R^{2} \mid x+r=y+r\right.$, some $\left.r \in I\right\}$ has $I$ as its only nontrivial class by the results of the last paragraph. All elements of $I$ are $\mu$-related to 0 , so $\mu=\Theta(I)$. This finishes the proof that the only nontrivial $\mu$-class is $0 / \mu$.

Our next goal is to prove that $|I|=2$. Since $I^{2} \subseteq I$ we get that $\Theta\left(I^{2}\right) \leq \Theta(I)$. We claim that $\Theta\left(I^{2}\right)<\Theta(I)$. Suppose otherwise that $\Theta\left(I^{2}\right)=\Theta(I)$. I is principal, since it is an annihilator ideal in a finite semiring, so let $a(>0)$ denote the largest element of $I$. Then $a^{2}$ is the largest element of $I^{2}$. But $I^{2}$ is a $\Theta\left(I^{2}\right)$-class, so if $\Theta\left(I^{2}\right)=\Theta(I)$, then $a^{2}$ is also the largest element of $I$. Hence, we get $a=a^{2}$ which is impossible since $a$ is a nonzero nilpotent element. Thus $0=\Theta\left(I^{2}\right)<\Theta(I)$, so $I^{2}=0$. Choose $u \in I$ such that $0 \prec u$ in the semilattice order. Now $\mu=\operatorname{Cg}(0, u)$ equals the equivalence relation on $R$ generated by the set of pairs $\left\{(p(0), p(u)) \mid p \in \operatorname{Pol}_{1} \mathbf{R}, p(0) \neq p(u)\right\}$. Choose $p(x) \in \operatorname{Pol}_{1} \mathbf{R}$. Using the semiring laws we may write $p(x)$ as $a_{n} x^{n}+\cdots+a_{1} x+a_{0}$. But since $I^{2}=0$, we get that $u^{k}=0^{k}=0$ for $k>1$. Hence, $(p(0), p(u))=(q(0), q(u))$ where $q(x)=a_{1} x+a_{0}$. Furthermore, since $0 \prec u$, we get that either $a_{1} u=0=a_{1} 0$ or $a_{1} u=u>0=a_{1} 0$. Hence, if $q(0) \neq q(u)$, then $(q(0), q(u))=(r(0), r(u))$ where $r(x)=x+a_{0}$. Now suppose that $u<a$ where $a$ is the largest element of $I$. Then since $(u, a) \in \operatorname{Cg}(0, u)$ we get a Mal'cev chain $u=m_{0}, m_{1}, \ldots, m_{l}=a$. Another Mal'cev chain is $u=m_{0}+u, m_{1}+u, \ldots, m_{l}+u=a$ so without loss of generality we may assume that the original chain consisted of distinct elements $\geq u$ and that $\left\{m_{i}, m_{i+1}\right\}$ $=\left\{r_{i}(0), r_{i}(u)\right\}$ for some $r_{i}(x)$ of the form $x+b_{i}=x+m_{i}$ or $x+m_{i+1}$. But for any such $r_{i}(x)$ we get that $r_{i}(0)=r_{i}(u)$ which contradicts the fact that the $m_{i}$ are distinct and $\left\{m_{i}, m_{i+1}\right\}=\left\{r_{i}(0), r_{i}(u)\right\}$. We conclude that $u$ is the top element of $I$. Since $0 \prec u$ we get that $I=\{0, u\}$. Relabel $u$ as $0^{\prime}$. Every element of $R-I$ is above $0^{\prime}$, so 0 is meet-irreducible in $\langle R ;+\rangle$. This proves the first part of $(i)$. We have also shown that the only nontrivial $\mu$-class is $\left\{0,0^{\prime}\right\}$. This proves (iii).

Let $J=\left\{r \in R \mid r \cdot 0^{\prime}=0\right\}$. $J$ is an annihilator ideal and $I \subseteq J \subseteq R-\{1\}$. We claim that $J=R-\{1\}$. Assume instead that $v \in R-\{1\}-J$. Then $\left(0,0^{\prime}\right) \in \operatorname{Cg}(1, v)$, so there is a unary polynomial $p(x)=a_{n} x^{n}+\cdots+a_{1} x+a+0$ such that $p(v) \neq p(1)$ and one of these elements equals 0 . Since $v<1$ and polynomials preserve order, this means that $p(v)=0<p(1)$. Write $p(x)=b_{n} x^{n}+\cdots+b_{1} x+b_{0}$. Since $v \notin J$ we get that $v \neq 0^{\prime}$, so $v=v^{2}$. Hence, $(p(v), p(1))=(q(v), q(1))$ where $q(x)=\left(b_{n}+\cdots+b_{1}\right) x+b_{0}$ $=c x+d$. Since $0=q(v)=c v+d$, we get that $d=0=c v$. Since $q(1)=c>0$, we get that $c \geq 0^{\prime}$. But now we have

$$
0=q(v)=c v=\left(c+0^{\prime}\right) v=c v+0^{\prime} v=v 0^{\prime} .
$$

But this implies that $v \in J$ contrary to our hypothesis. Our assumption that there is a 
$v \in R-\{1\}-J$ is false and so $J=R-\{1\}$. This proves (iv). Since $J$ is closed under + we have proved that 1 is join-irreducible in $\langle R ;+\rangle$. This finishes the proof of $(i)$.

We can finish this lemma by showing that $\langle R-\{0,1\} ;+\rangle \cong\langle\{0,1\} ;+\rangle^{n}$ for some $n$. This holds with $n=0$ if $R=\left\{0,0^{\prime}, 1\right\}$, so assume that $R \neq\left\{0,0^{\prime}, 1\right\}$. Let $1^{\prime}$ be the largest element of $R-\{1\}$. Since the ordering on $R-\{0,1\}$ is that of a distributive lattice with more than one element, we only need to show that $1^{\prime}$ is the join of the covers of $0^{\prime}$. Set $w=\bigvee\left\{y \in R \mid 0^{\prime} \prec y\right\}$. Our assumption that $R \neq\left\{0,0^{\prime}, 1\right\}$ guarantees that $w \leq 1^{\prime}$ Assume that $w<1^{\prime}$. Then $\left(0,0^{\prime}\right) \in \operatorname{Cg}\left(w, 1^{\prime}\right)$, so there is a polynomial $p(x)=f_{n} x^{n}+\cdots+f_{0}$ such that $p(w)=0<p\left(1^{\prime}\right)$. As in above arguments we may assume that $p(x)=g x+h$. Since $0=p(w)=g w+h$ we get that $h=0$. Hence, there is an element $g \in R$ such that $g w=0<g 1^{\prime}$. Since $0<g 1^{\prime}$, we get that $0<g$. Since $0^{\prime} 1^{\prime}=0<g 1^{\prime}$, we get that $0^{\prime} \neq g$. Hence $0^{\prime}<g$. It follows that there is some $y$ such that $0^{\prime} \prec y \leq g$. But now $y \leq w, y \leq g$ and $y=y y \leq g w=0$. This is a contradiction, concluding the proof of $(i i)$.

The conditions enumerated in Lemma 3.9 determine minimal semirings up to isomorphism; there is one isomorphism type for each $n$ that appears in Lemma 3.9 (ii). Conditions $(i)$ and $(i i)$ of Lemma 3.9 determine the +-ordering on the semiring. Conditions (iii) and (iv) and the fact that $\mathbf{R} / \mu \models r^{2}=r$ imply that, except for $1 \cdot 0^{\prime}=0^{\prime} \cdot 1=0^{\prime}$, no product of two elements equals $0^{\prime}$. Thus, all products which do not involve $0^{\prime}$ are determined by the order while all products involving $0^{\prime}$ are determined by Lemma $3.9(i v)$.

LEMMA 3.10 Assume that $\mathcal{U}$ is a locally finite variety of semilattice modes and that $\mathbf{R}(\mathcal{U})$ is minimal. Then $\mathcal{U}$ contains a subdirectly irreducible mode $\mathbf{A}$ with least element 0 and second least element $u$ and the following properties hold:

(i) The largest element $t \in A$ is join-irreducible and $u<t$

(ii) If $t_{*} \prec t$ in $\langle A ;+\rangle$, then $\left\{t_{*}, t\right\}$ is a subuniverse of $A$.

(iii) $\operatorname{Sg}^{\mathbf{A}}\left(\left\{t_{*}, t\right\}\right) \cong \operatorname{Sg}^{\mathbf{A}}(\{0, u\})$.

Proof: Since $\mathbf{R}(\mathcal{U})$ is subdirectly irreducible, $\mathcal{U}$ is a join-irreducible element of the lattice of subvarieties of $\mathcal{U}$ by Theorem 2.7. If the lower cover of $\mathcal{U}$ is $\mathcal{U}_{*}$, then choose a subdirectly irreducible $\mathbf{A} \in \mathcal{U}-\mathcal{U}_{*}$. We shall argue that $\mathbf{A}$ has the desired properties.

Since $\mathcal{U}=\mathrm{V}(\mathbf{A})$, we get that $\mathbf{A} \cong \mathcal{I}(\mathcal{U})$. Therefore, the order of $\langle A ;+\rangle$ is the same as the order of $\langle\mathcal{I}(\mathcal{U}) ; \oplus\rangle$. The semilattice operation on $\mathcal{I}(\mathcal{U})$ is ideal intersection. Each ideal is principal, so this operation corresponds to the meet in $\langle R(\mathcal{U}) ;+\rangle$. As join-semilattices, we find that the order on $\mathbf{A}$ is the same as the order on $\mathcal{I}(\mathcal{U})$, but it is dual to the order on $\mathbf{R}(\mathcal{U})$. The order-theoretic statements deduced about minimal semirings in Lemma 3.9 allow us to deduce that $\langle A ;+\rangle$ has a largest element $t$ which is join-irreducible, a least element 0 which is meet-irreducible, at least one other element, and $\langle A-\{0, t\} ;+\rangle$ is a Boolean semilattice. This is more than enough to deduce part (i) of this lemma. If $t_{*}$ is the lower cover of $t$, then the unary polynomial $p(x)=$ $x+t_{*}$ is an endomorphism with image $\left\{t_{*}, t\right\}$, so this set is a subuniverse. In order to deduce that $\operatorname{Sg}^{\mathbf{A}}\left(\left\{t_{*}, t\right\}\right) \cong \operatorname{Sg}^{\mathbf{A}}(\{0, u\})$, we may identify $\mathbf{A}$ with $\mathcal{I}(\mathcal{U})$. Under this identification the reader will verify that the top element of $\mathcal{I}(\mathcal{U})$ is the annihilator ideal $\{0\}=t$, the lower cover of this ideal is $\left\{0,0^{\prime}\right\}=t^{*}$, the least element is the annihilator 
ideal $R=R(\mathcal{U})=0^{\mathbf{A}}$ and the upper cover of $R$ is $R-\{1\}=u^{\mathbf{A}}$. The two subuniverses we are interested in are the subuniverses $\{R, R-\{1\}\}$ and $\left\{\left\{0,0^{\prime}\right\},\{0\}\right\}$ of $\mathcal{I}(\mathcal{U})$.

The algebra $\mathcal{I}(\mathcal{U}) \cong \mathbf{A}$ is subdirectly irreducible; so, by Theorem 2.3, it is term equivalent to the algebra on the same base set with basic operations $r^{-1}(x) \oplus y, r \in$ $R(\mathcal{U})$. Changing to a term equivalent algebra does not affect isomorphisms between subalgebras, so we may assume that the basic operations of $\mathcal{I}(\mathcal{U})$ are of the form $r^{-1}(x) \oplus y=r^{-1}(x) \cap y, r \in R(\mathcal{U})$. We must show that any such operation behaves the same way on $\{R, R-\{1\}\}$ and $\left\{\left\{0,0^{\prime}\right\},\{0\}\right\}$. If $r \neq 1$, then $r^{-1}(R-\{1\})=R$ and from Lemma 3.9 we get that $r^{-1}(\{0\}) \supseteq\left\{0,0^{\prime}\right\}$. Hence, $r^{-1}(x) \cap y=y$ on each of these subuniverses. If $r=1$, then $r^{-1}(x) \cap y=x \cap y$ on each of these subuniverses. Clearly the bijection $\{0\} \mapsto R-\{1\},\left\{0,0^{\prime}\right\} \mapsto R$ preserves all operations of the form $r^{-1}(x) \oplus y$, so $($ iii $)$ is established.

THEOREM 3.11 If $\mathcal{V}$ is a locally finite variety of semilattice modes, then $\mathcal{V}$ has the $A P$ if and only if $\mathbf{R}(\mathcal{V})$ is a bounded distributive lattice.

Proof: The "if" direction was proved in Theorem 3.7. We must prove that if $\mathcal{V}$ is locally finite and $\mathbf{R}(\mathcal{V})$ is not a bounded distributive lattice, then $\mathcal{V}$ does not have the AP.

Assuming that $\mathbf{R}(\mathcal{V})$ is not a bounded distributive lattice, choose $\mathcal{U} \subseteq \mathcal{V}$ such that $\mathbf{R}(\mathcal{U})$ is minimal. Then $\mathcal{U}$ contains a subdirectly irreducible algebra $\mathbf{B}$ with the properties listed in Lemma 3.10. $\mathbf{B} \in \mathcal{V}$, so we will finish the proof by showing that $\mathbf{B}$ is contained in no locally finite variety of modes which has the AP. This will finish the proof.

Let $\mathbf{A}$ be the subalgebra of $\mathbf{B}$ with universe $\{0, u\}$. If $t$ is the top element of $\mathbf{B}$ and $t_{*}$ is the lower cover of $t$, then $0 \prec u \leq t_{*} \prec t$ and $\left\{t_{*}, t\right\}$ is a subuniverse of $\mathbf{B}$. The map $f_{1}$ defined by $0 \mapsto t_{*}, u \mapsto t$ is an isomorphism from $\mathbf{A}$ onto the subalgebra on $\left\{t_{*}, t\right\}$. If a variety with the $\mathrm{AP}$ contains $\mathbf{B}$, then it contains $\mathbf{A}$ and there must be a way to complete the amalgam $\left(\mathbf{A} ; f_{1}, \mathbf{B} ; g_{1}, \mathbf{B}\right)$ where $g_{1}: \mathbf{A} \rightarrow \mathbf{B}$ is inclusion. Since $\mathbf{B}$ is subdirectly irreducible and $g_{1}$ is essential, we must be able to complete $\left(\mathbf{A} ; f_{1}, \mathbf{B} ; g_{1}, \mathbf{B}\right)$ with a triple $\left(f_{2} ; g_{2} ; \mathbf{D}\right)$ where $\mathbf{D}$ is subdirectly irreducible and $f_{2}$ is essential. Of course this means that $\mathbf{D}$ contains elements

$f_{2}(0)<f_{2}(u) \leq f_{2}\left(t_{*}\right)=f_{2} \circ f_{1}(0)=g_{2} \circ g_{1}(0)=g_{2}(0)<f_{2}(t)=g_{2}(u) \leq g_{2}\left(t_{*}\right)<g_{2}(t)$.

The composition $f_{2} \circ g_{1}: \mathbf{A} \rightarrow \mathbf{D}$ is essential because each function is. We have another embedding $g_{2} \circ f_{1}: \mathbf{A} \rightarrow \mathbf{D}$. It makes sense now to consider the amalgam $\left(\mathbf{A} ; g_{2} \circ f_{1}, \mathbf{D} ; f_{2} \circ g_{1}, \mathbf{D}\right)$. $\mathbf{D}$ is subdirectly irreducible and $f_{2} \circ g_{1}$ is essential. We are back in the same situation we were in before, but now we have $\mathbf{D}$ in place of $\mathbf{B}$. This is an improvement since $\mathbf{D}$ is larger than $\mathbf{B}$. We know this because $g_{2}: \mathbf{B} \rightarrow \mathbf{D}$ is an embedding which is not onto. In fact every element of $g_{2}(B)$ is $\geq g_{2}(0)$ which is strictly larger than $f_{2}(0)$. Since this argument can be repeated as often as we like, we find that any variety which contains $\mathbf{B}$ and has the AP cannot have a finite bound on the size of its subdirectly irreducibles. Hence, it cannot be locally finite by Corollary 2.5. By our remarks in the second paragraph this completes the proof.

We have proved that if $\mathcal{V}$ is any variety of modes where $\mathbf{R}(\mathcal{V})$ is a bounded distributive lattice, then $\mathcal{V}$ has the AP. Conversely, we have shown that if $\mathcal{V}$ has the 
AP and has a subvariety $\mathcal{U}$ where $\mathbf{R}(\mathcal{U})$ is minimal, then $\mathcal{V}$ cannot be locally finite (equivalently, $|R(\mathcal{V})|$ cannot be finite). One can actually show that if $\mathcal{V}$ has the AP and has a subvariety $\mathcal{U}$ where $\mathbf{R}(\mathcal{U})$ is minimal, then $\mathbf{R}(\mathcal{V})$ cannot be locally finite. (Equivalently, $\mathbf{R}(\mathcal{V})$ has an element of infinite order.) At this point it is natural to wonder if the hypothesis that " $\mathcal{V}$ is a locally finite variety of semilattice modes" in Theorem 3.11 can be weakened to the hypothesis that " $\mathcal{V}$ is a variety of semilattice modes where $\mathbf{R}(\mathcal{V})$ is locally finite." One cannot use the same types of arguments that we have used above since, as the next example shows, such a $\mathcal{V}$ may have no subvariety $\mathcal{U}$ where $\mathbf{R}(\mathcal{U})$ is minimal.

Example 2. This example is of a locally finite, commutative semiring $\mathbf{R}$ satisfying $1+r=1$, which has no homomorphic image that is minimal in any sense resembling our use of the term in Lemma 3.9. In fact, if $\mathbf{S}$ is any homomorphic image of $\mathbf{R}$ which is not isomorphic to the 1-element or 2-element lattice, then $\mathbf{R}$ is also a homomorphic image of $\mathbf{S}$ !

The universe of $\mathbf{R}$ is the unit interval of the real numbers, $[0,1]$. We define $0^{\mathbf{R}}=$ $0,1^{\mathbf{R}}=1, x+y=\max \{x, y\}$ and $x \cdot y=\max \{x+y-1,0\}$. We leave it to the reader to verify the semiring laws along with commutativity of multiplication and $1+r=1$.

If $z \in R$ and $z<1$, then $z<1-\frac{1}{n}$ for some $n$. Since $0 \leq z^{n} \leq\left(1-\frac{1}{n}\right)^{n}=0$, we get that every element of $R-\{1\}$ is nilpotent. Choose any $x, y \in R$ with $x<y$ and set $z=x-y+1$. One checks that $x \leq z<1$ and $y \cdot z=x$. Hence $(y \cdot z, y) \in \operatorname{Cg}(x, y)$. From this we get that $\left(y z^{n+1}, y z^{n}\right) \in \operatorname{Cg}(x, y)$, so $\left(y z^{n+1}, y\right) \in \operatorname{Cg}(x, y)$ for all $n$. But as we observed, $z<1$ implies that $z$ is nilpotent. Hence $(0, y) \in \operatorname{Cg}(x, y)$. If $0 \leq w \leq y$, then $(w, y)=(w+0, w+y) \in \operatorname{Cg}(x, y)$, too. Altogether we have that $\operatorname{Cg}(x, y)=\Theta([0, y])$. Hence, every congruence on $\mathbf{R}$ is an ideal congruence. The compact congruences are those of the form $\Theta([0, y])$ while the non-compact congruences are those of the form $\Theta([0, y))$. For a fixed $y<1$ the function

$$
f: \mathbf{R} \rightarrow \mathbf{R}: x \mapsto \max \left\{\frac{x-y}{1-y}, 0\right\}
$$

is a surjective endomorphism with kernel $\Theta([0, y])$. Hence, if there is a surjection $g: \mathbf{R} \rightarrow \mathbf{S}$ with ker $g=\Theta=\Theta([0, z])$ or $\Theta([0, z)), z<1$, then we may choose $y \in[z, 1)$ and get

$$
\mathbf{R} \cong \mathbf{R} / \Theta([0, y]) \cong(\mathbf{R} / \Theta) /(\Theta([0, y]) / \Theta) .
$$

The last semiring is a homomorphic image of $\mathbf{S}$. Hence, if $\mathbf{R}$ has a homomorphism onto $\mathbf{S}$ with kernel $\Theta([0, z])$ or $\Theta([0, z)), z<1$, then $\mathbf{S}$ has a homomorphism onto $\mathbf{R}$. (If $\mathbf{R}$ has a homomorphism onto $\mathbf{S}$ with kernel $\Theta([0,1])$ or $\Theta([0,1))$, then $\mathbf{S}$ is the 1-element or 2-element lattice, respectively.)

A natural question now is whether there is any variety of semilattice modes which has the AP where $\mathbf{R}(\mathcal{V})$ is not a bounded distributive lattice. There are such varieties and the next example describes one of them.

Example 3. We will describe a mode which generates a variety with the AP even though the semiring of the variety is not a bounded distributive lattice. The mode will be of the form $\mathbf{S}(U)$ where $\mathbf{S}$ is the ordinal $\omega+1$ with the join operation defined as follows: for $\alpha, \beta<\omega+1$ define $\alpha+\beta=\max \{\alpha, \beta\}$. $U$ will be the monoid of 
endomorphisms of $\mathbf{S}$ generated by the following endomorphism: for $0<\alpha<\omega$ define $f(\alpha)=\alpha-1$ and define $f(0)=0, f(\omega)=\omega$. Thus, $\mathbf{A}=\mathbf{S}(U)$ has basic operations $F_{n}(x, y)=f^{n}(x)+y, 0 \leq n<\omega$. Note that, since $\mathbf{S}$ and $U$ satisfy the conditions enumerated in Example 1, $\mathbf{A}$ is subdirectly irreducible.

It is shown in Theorem 4.13 of [3] that, when $\mathbf{A}$ is subdirectly irreducible with least element 0 ,

$$
\mathbf{R}(\mathrm{V}(\mathbf{A})) \cong\left\langle U ; \circ,+, i d_{A}, 0\right\rangle .
$$

Hence, we may think of $\mathbf{R}=\mathbf{R}(\mathrm{V}(\mathbf{A}))$ as consisting of the elements $0<\cdots \prec f^{2} \prec f \prec$ $1=i d_{A}$. From this one gets that $\mathbf{R}$ has an underlying semilattice dually isomorphic to that of $\mathbf{A}$. Recall that $\mathcal{I}(\mathrm{V}(\mathbf{A}))$ is a mode constructed from the annihilator ideals of $\mathbf{R}$. These ideals are of the form $\{0\}$ or $\left(f^{n}\right], 0 \leq n<\omega$, so $\mathcal{I}(\mathbf{V}(\mathbf{A}))$ has an underlying semilattice isomorphic to the underlying semilattice of $\mathbf{A}$ and has basic operations

$$
\left[F_{n}\right](x, y)=\left(\widehat{F_{n}}\right)_{0}^{-1}(x) \oplus\left(\widehat{F_{n}}\right)_{1}^{-1}(y)=\left(f^{n}\right)^{-1}(x) \cap y .
$$

If $I$ is the order-ideal $\left(f^{n}\right]$, then $f^{-1}(I)=\left(f^{n-1}\right]$ and $\left(f^{n-1}\right] \prec\left(f^{n}\right]$ in $\mathcal{I}(\mathrm{V}(\mathbf{A}))$. This computation shows that $f^{-1}$ acts on the underlying semilattice of $\mathcal{I}(\mathrm{V}(\mathbf{A}))$ in the same way that $f$ acts on the underlying semilattice of $\mathbf{A}$. Thus, $\left[F_{n}\right]$ acts on $\mathcal{I}(\mathrm{V}(\mathbf{A}))$ in the same way that $F_{n}$ acts on $\mathbf{A}$. It follows that $\mathbf{A}$ is isomorphic to $\mathcal{I}(\mathrm{V}(\mathbf{A}))$.

Theorem 2.4 proves that any subdirectly irreducible in $\mathrm{V}(\mathbf{A})$ embeds into $\mathcal{I}(\mathrm{V}(\mathbf{A}))$, so every subdirectly irreducible in $\mathbf{V}(\mathbf{A})$ is isomorphic to a subalgebra of $\mathbf{A}$. Assume $\mathbf{C} \leq \mathbf{A}$. Let $c_{0}$ be the least element of $\mathbf{A}$ which lies in $\mathbf{C}$. For $c<\omega$ equal to any element of $C-\left\{c_{0}\right\}$ it is possible to choose $n$ and evaluate the operation $F_{n}\left(c, c_{0}\right)$ so as to obtain any element of $\mathbf{A}$ in the interval $\left[c_{0}, c\right]$. It follows that $C \cap[0, \omega)$ is an interval in $\langle A ; \leq\rangle$; hence $C$ is of the form $\left[c_{0}, a\right)$ for some $a \in A$ or $C$ is of the form $\left[c_{0}, a\right) \cup\{\omega\}$ for some $a \in A$. Conversely, any subset $C \subseteq A$ of the form $\left[c_{0}, a\right)$ or $\left[c_{0}, a\right) \cup\{\omega\}$ is a subuniverse. It is straightforward to use the operations $\left\{F_{n} \mid n<\omega\right\}$ to prove that any such $\mathbf{C}$ is subdirectly irreducible if it has at least two elements. To summarize, we have shown in this paragraph that the subdirectly irreducible algebras in $\mathrm{V}(\mathbf{A})$ are precisely the algebras isomorphic to nontrivial subalgebras of $\mathbf{A}$.

To show that $\mathrm{V}(\mathbf{A})$ has the AP, we must show how to complete every amalgam of the form $\left(\mathbf{D} ; g_{1}, \mathbf{M} ; h_{1}, \mathbf{N}\right)$ where $\mathbf{D}$ has more than one element, $\mathbf{M}$ is subdirectly irreducible and $h_{1}$ is essential. But if $\mathbf{M}$ is subdirectly irreducible, then there is an embedding $i: \mathbf{M} \rightarrow \mathbf{A}$ and if $\left(g_{2} ; h_{2} ; \mathbf{P}\right)$ completes $\left(\mathbf{D} ; i \circ g_{1}, \mathbf{A} ; h_{1}, \mathbf{N}\right)$, then $\left(g_{2} \circ i ; h_{2} ; \mathbf{P}\right)$ completes $\left(\mathbf{D} ; g_{1}, \mathbf{M} ; h_{1}, \mathbf{N}\right)$. Hence, we only need to consider those amalgams $\left(\mathbf{D} ; g_{1}, \mathbf{M} ; h_{1}, \mathbf{N}\right)$ where $\mathbf{M}=\mathbf{A}$. $\mathbf{A}$ is hereditarily subdirectly irreducible and $\mathbf{D}$ is isomorphic to a subalgebra of $\mathbf{A}$, so we get that $\mathbf{D}$ is subdirectly irreducible. $\mathbf{N}$ is an essential extension of $\mathbf{D}$, so $\mathbf{N}$ is subdirectly irreducible as well. There is an embedding $j: \mathbf{N} \rightarrow \mathbf{A}$ and if we can complete $\left(\mathbf{D} ; g_{1}, \mathbf{A} ; j \circ h_{1}, \mathbf{A}\right)$ with $\left(g_{2} ; h_{2} ; \mathbf{P}\right)$, then $\left(g_{2} \circ j ; h_{2} ; \mathbf{P}\right)$ completes $\left(\mathbf{D} ; g_{1}, \mathbf{A} ; h_{1}, \mathbf{N}\right)$. Hence, we only need to show how to complete those amalgams of the form $\left(\mathbf{D} ; g_{1}, \mathbf{A} ; h_{1}, \mathbf{A}\right)$. We may assume that $g_{1}$ is inclusion, so that $D \subseteq A$.

Let $d_{0}$ be the least element of $D$. Since $\mathbf{D}$ has more than one element, $d_{0}<\omega$. From our above descriptions we see that $\mathbf{D}$ must belong to one of only four families of subalgebras of $\mathbf{A}$ :

(i) $D=\left[d_{0}, \omega\right)$ for some $d_{0}<\omega$,

(ii) $D=\left[d_{0}, \omega\right]$ for some $d_{0}<\omega$,

$(\text { iii })_{n} \quad D=\left[d_{0}, d_{0}+n\right]$ for some $d_{0}<d_{n}<\omega$ or 
$(i v)_{n} \quad D=\left[d_{0}, d_{0}+n\right] \cup\{\omega\}$ for some $d_{0} \leq d_{n}<\omega$.

No subalgebra of $\mathbf{A}$ in one of these families is isomorphic to a subalgebra from a different family. Furthermore, no subalgebra of type $(i i i)_{i}$ is isomorphic to one of the form $(i i i)_{j}$ if $i \neq j$ (and the same for $(i v)_{i}$ and $(i v)_{j}$.) The subalgebras from the first family may be distinguished from those in other families because they are infinite, but have no last element. The second, because they are infinite, but have a last element. The third, because they are finite, have $n$ elements and $F_{1}\left(l, d_{0}\right) \neq l$ for $l=$ the last element of $D$. The fourth, because they are finite, have $n+1$ elements and $F_{1}\left(l, d_{0}\right)=l$ for $l=$ the last element of $D$. One can show, case by case, that for any $\mathbf{D}$ belonging to one of the four families it is possible to complete any amalgam of the form $\left(\mathbf{D} ; g_{1}, \mathbf{A} ; h_{1}, \mathbf{A}\right)$. The arguments are nearly identical for all cases, so we shall only write down the argument for the case where $\mathbf{D}$ is in family $(i v)$.

Assume that we have an amalgam $\left(\mathbf{D} ; g_{1}, \mathbf{A} ; h_{1}, \mathbf{A}\right)$ where $\mathbf{D}$ belongs to family $(i v)$ and let $a=g_{1}\left(d_{0}\right)$ and $b=h_{1}\left(d_{0}\right)$. Define $g_{2}: \mathbf{A} \rightarrow \mathbf{A}$ by $g_{2}(x)=b+x$. Define $h_{2}: \mathbf{A} \rightarrow \mathbf{A}$ by $h_{2}(x)=a+x$. (In these last two sentences only, the symbol + denotes ordinal addition.) Then $\left(g_{2} ; h_{2} ; \mathbf{A}\right)$ completes $\left(\mathbf{D} ; g_{1}, \mathbf{A} ; h_{1}, \mathbf{A}\right)$. By Lemma 3.1, we conclude that $\mathrm{V}(\mathbf{A})$ has the $\mathrm{AP} . \mathbf{R}(\mathrm{V}(\mathbf{A}))$ is not a bounded distributive lattice. (It turns out that $\mathbf{R}(\mathrm{V}(\mathbf{A}))$ is the free 1-generated commutative semiring satisfying $1+r=1$.)

We end this paper with a question and a problem, both suggested by the results of this section.

Question 1. Are there varieties $\mathcal{U}$ and $\mathcal{V}$ with $\mathbf{R}(\mathcal{U}) \cong \mathbf{R}(\mathcal{V})$ where exactly one of them has the AP?

Assuming that the answer to the previous question is "No",

Problem 2. Describe those $\mathbf{R}(\mathcal{V})$ for which $\mathcal{V}$ has the AP.

Certain special cases might be considered first. For example, one might consider only when $\mathbf{R}(\mathcal{V})$ is finitely generated or only when $\mathbf{R}(\mathcal{V})$ is linearly ordered.

\section{References}

[1] C. C. Chang and H. J. Keisler, Model Theory, 3rd ed., Studies in Logic v. 73, North Holland, 1990.

[2] K. Kearnes, Finite algebras that generate an injectively complete modular variety, Bull. Austral. Math. Soc. 44 (1991), 303-324.

[3] K. Kearnes, Semilattice Modes I: the associated semiring, Algebra Universalis

[4] K. Kearnes, The structure of finite modes, in progress.

[5] R. McKenzie, G. McNulty and W. Taylor, Algebras, Lattices and Varieties, vol. 1, Wadsworth \& Brooks/Cole, 1987.

[6] A. Romanowska and J.D.H. Smith, Modal Theory - an Algebraic Approach to Order, Geometry and Convexity, Heldermann Verlag, Berlin, 1985. 